\title{
Red and blue light treatments of ripening bilberry fruits reveal differences in signaling through ABA regulated anthocyanin biosynthesis
}

\author{
Amos Samkumar ${ }^{1}$, Dan Jones ${ }^{2}$, Katja Karppinen ${ }^{1}$, Andrew Dare ${ }^{3}$, Nina Sipari ${ }^{4}$, Richard \\ Espley $^{5}$, Inger Martinussen ${ }^{6}$, and Laura Jaakola ${ }^{7}$ \\ ${ }^{1}$ UiT The Arctic University of Norway \\ ${ }^{2}$ New Zealand Institute for Plant and Food Research Ltd \\ ${ }^{3}$ The New Zealand Institute for Plant and Food Research \\ ${ }^{4}$ University of Helsinki \\ ${ }^{5}$ Plant \& Food Research \\ ${ }^{6}$ Norwegian Institute of Bioeconomy Research \\ ${ }^{7}$ UIT The Arctic University of Norway
}

May 29, 2021

\begin{abstract}
The biosynthesis of anthocyanins has been shown to be influenced by light quality. However, the molecular mechanisms underlying the light-mediated regulation of fruit anthocyanin biosynthesis are not well understood. In this study, we analyzed the effects of supplemental red and blue light on the anthocyanin biosynthesis in non-climacteric bilberry (Vaccinium myrtillus L.). After six days of continuous irradiation during fruit ripening, both red and blue light elevated concentration of total anthocyanins, but especially red light promoted accumulation of delphinidins. Transcriptomic analysis of ripening berries showed that both light treatments up-regulated all the major anthocyanin structural genes, the key regulatory MYB transcription factors and abscisic acid (ABA) biosynthetic genes. However, higher induction of specific genes of anthocyanin and delphinidin biosynthesis alongside ABA signal perception and metabolism were found in red light. The difference in red and blue light signaling was found in NCED, ABA receptor PYL and catabolic ABA-8'hydroxylase gene expression. Red light also upregulated expression of SNARE domain transporters, which may indicate involvement of these proteins in vesicular trafficking of anthocyanins during fruit ripening. Our results suggest differential signal transduction and transport mechanisms between red and blue light in in ABA- regulated anthocyanin and delphinidin biosynthesis during non-climacteric fruit ripening.
\end{abstract}

\section{Introduction}

Light is among the most important environmental factors modifying plant growth and development. Higher plants have evolved sophisticated mechanisms to perceive light signals through specialized photoreceptors that respond to various light properties, such as light intensity, light spectral quality, and photoperiod (Briggs \& Olney, 2001; Zoratti et al., 2014a). Different visible light photoreceptors and UV-B receptors can sense light signals from a broad range of solar spectrum between $280 \mathrm{~nm}$ to $750 \mathrm{~nm}$ (Möglich et al., 2010). Most of the transducible wavelengths absorbed by plants fall within 400-700 nm (from blue to red), which is also commonly referred to as photosynthetically active radiation (PAR; Chen et al., 2004). Within this range, phytochrome B has a specialized function towards red light while cryptochrome sense blue light to promote photomorphogenesis (Li \& Yang 2007; Lu et al., 2015).

After light perception, phytochrome and cryptochrome interact with the E3 ubiquitin ligase constitutive 
photomorphogenesis protein 1 (COP1), which is the key light signaling regulator (Lau et al., 2012). In the dark, COP1 directly interacts and represses the action of elongated hypocotyl (HY5) gene inhibiting light signal transmittance and circadian clock genes, also the flowering regulators, such as CONSTANS (CO) via the proteasomal degradation complex (Zoratti et al., 2014a; Bhatnagar et al., 2020). Under light, COP1 activity is repressed allowing the expression of $H 5$ and positive transcriptional regulators in a number of developmental processes and metabolic pathways, including anthocyanin biosynthesis (Wu et al., 2019).

Light quality has a significant influence on plant secondary metabolism (Ouzounis et al., 2015). For example, biosynthesis of polyphenols, anthocyanins, glucosinolates, terpenes, and carotenoids in plant tissues are responsive to light quality and they have important roles for example in photoprotection (Ballaré, 2014; Holopainen et al., 2018). Light quality also influences the metabolite accumulation in fruits and berries, as shown by several pre- and postharvest light treatments in different fruit crops (Koyama et al., 2012; Tao et al., 2018; Kokalj et al., 2019). High light intensity has generally been reported to increase anthocyanin accumulation in fruits, but it is also affected by light quality (Jaakola et al., 2013; Ma et al., 2019). For instance, red light has been reported to increase anthocyanin content in strawberry (Fragaria $x$ ananassa) , and blue light radiation increased anthocyanin levels after selective bagging treatment in pear (Pyrus communis L.) fruit (Miao et al., 2016; Tao et al., 2018). In bilberry, Zoratti et al., (2014b) showed that short term treatment with supplemental monochromatic light affected the anthocyanin profile during bilberry fruit development. However, the regulatory mechanisms behind the effect of red and blue light wavelengths on anthocyanin biosynthesis are not well understood.

Anthocyanins are prominent phenolic compounds in plants that are biosynthesized from the well-studied flavonoid pathway, which branches from phenylpropanoid biosynthesis (Tohge et al., 2017). The major early biosynthetic enzymes involved in flavonoid biosynthesis are phenylalanine ammonia lyase (PAL), chalcone synthase (CHS), and chalcone isomerase (CHI). At the branchpoint of flavonoid biosynthesis, flavonoid 3' hydroxylase (F3'H) and flavonoid 3'5' hydroxylase (F3'5'H) direct biosynthesis to cyanidin and delphinidin compounds, respectively (Jaakola et al., 2002). The six major anthocyanin aglycone end-products, namely cyanidin, delphinidin, pelargonidin, petunidin, malvidin, and peonidin, are biosynthesized by the late biosynthetic enzymes dihydroflavonol 4-reductase (DFR) and anthocyanin synthase (ANS) and further glycosylated by UDP-glucose: flavonoid- $O$-glycosyltransferase (UFGT) as the last step in anthocyanin biosynthesis (Wu et al., 2017). Anthocyanins are transported to the vacuole after their biosynthesis. The mechanisms of anthocyanin transport are not fully understood but common transporter proteins, such as ATP-binding cassettes (ABCs), multidrug and toxic extrusion (MATEs) and glutathione S-transferases (GSTs), are commonly believed to be responsible for transportation to vacuolar membrane and lumen (Behrens et al., 2019). Another proposed model has been vesicular trafficking by phagosomes involving engulfment of anthocyanin bodies by endosomes before reaching the vacuole (Chanoca et al., 2015). The vesicular transportation is mediated by SNARE (soluble $N$-ethylmaleimide-sensitive fusion protein attachment protein receptors) protein complexes, which are proposed to have a role in cellular transport in higher plants under stress responses (Pečenková et al., 2017).

The biosynthesis of flavonoids is directly controlled by the transcriptional regulatory MBW complex, consisting of MYB (myeloblastosis), bHLH (basic helix-loop-helix) transcription factors and WD-40 repeat proteins (Feller et al., 2011; Xu et al., 2015). R2R3 MYB transcription factors (TFs) are known as the key regulators of anthocyanin biosynthesis and are responsive to shifts in light spectral quality (Zoratti et al., 2014a). In grapes, two R2R3 MYB TFs, $V v M Y B A 1$ and $V v M Y B A 2$, controlling anthocyanin biosynthesis specifically regulate UFGT (Walker et al., 2007). In apple and peach, R2R3 MYBA-type TFs activate anthocyanin biosynthesis by interacting with both the UFGT and DFR promoters during fruit ripening (Takos et al., 2006; Ravaglia et al., 2013).

In contrast to climacteric fruits, the ripening process of non-climacteric fruits is independent of respiratory burst and ethylene accumulation (Cherian et al., 2014). Instead, abscisic acid (ABA), which is synthesized by the key cleavage enzyme 9-cis-epoxycarotenoid dioxygenase (NCED) in apocarotenoid pathway, has been shown to be a major regulator of ripening in non-climacteric fruits, such as strawberry, grapes and bilberry 
(Jia et al., 2011; Ferrera et al., 2015; Karppinen et al., 2018). The ABA signal transduction is known to be mediated by pyrabactin resistance/like receptors (PYR/PYL) and ABA responsive element binding factors (ABFs) through SQUAMOSA-MADS box (TDR-type) transcription factors leading to regulation of the MBW complex proteins (Chung et al., 2019). Another model has been proposed illustrating that ABA interacts directly with PYR by inhibiting type2C protein phosphatases subsequently binding with ABFs and transduces the ABA signaling pathway (Park et al., 2009).

Bilberry (Vaccinium myrtillus L.), also known as European blueberry, is one of the most important wild perennial berry species of Vaccinium genus and predominantly found in Northern Europe (Chu et al., 2011; Karppinen et al. 2016a; Zoratti, et al., 2016). The species has gained global interest due to its abundant health-beneficial bioactive compounds, including phenolic compounds, carotenoids, vitamins but especially anthocyanins, which constitutes $90 \%$ of total phenolics in these berries and give distinct deep blue color to both skin and flesh (Karppinen et al., 2016a). Several studies have reported consumption of bilberries to reduce risk of metabolic syndrome and various microbial and degenerative diseases (Chu et al., 2011; Nile \& Park, 2014; Bujor et al., 2016). In bilberry, delphinidin and cyanidin glycosides are the major anthocyanins followed by malvidin and petunidin glycosides (Müller et al., 2012; Zoratti et al., 2016; Thornthwaite et al., 2020). In particular, delphinidins, which are abundant in northern clones alongside malvidins, have been recently linked to many biological and health-beneficial activities (Nakaoga et al., 2019; Heysieattalab \& Sadeghi, 2020).

In the present study, we utilized the Illumina-based RNA-seq approach to produce transcriptome libraries from ripening bilberry fruit grown under supplemental red and blue light conditions. We specifically focused on the differences in red and blue light signal transduction in regulation of anthocyanin biosynthesis. The red and blue light emitting diodes (LEDs) used in our study give an opportunity to provide high intensity spectral wavelengths to plants as source of light for studying the effect of light quality to biosynthesis of phytochemicals.

\section{Materials and methods}

\subsection{Plant material and light treatments}

Wild bilberry (Vaccinium myrtillus L.) ecotype from Troms $\varnothing$, Norway $\left(69^{\circ} 75^{\prime} \mathrm{N}, 19 \mathrm{deg} 01^{\prime} \mathrm{E}\right)$ was used for the experiments. The bilberry bushes were collected during early July after the fruit set at stage S2 when berries were small, unripe, and green (Figure1a). The plants were collected in boxes $(50 \times 70 \mathrm{~cm})$ with their root system and forest soil and watered well. The plants were kept in the phytotron conditions at 16degC for few days to acclimatize until they reached berry developmental stage S3 when berries were large, unripe, and green (Figure 1a)

For light treatments, the plants were placed in chambers covered from sides with photo reflective sheets and irradiated from top with blue $(460 \mathrm{~nm})$ or red $(660 \mathrm{~nm})$ light wavelength provided by the Heliospectra RX30 lamps (Heliopspectra AB, Gothenburg, Sweden; Figure 1b). In addition to the specific spectral light wavelengths, the plants received continuous ambient white light $(400-800 \mathrm{~nm})$ from the top. The plants under the ambient white light $(400-800 \mathrm{~nm})$ served as control for the experiment. All plants were kept at $16 \mathrm{degC}$, and the photon fluence rate $\left(\mu \mathrm{mol} \mathrm{m} \mathrm{m}^{-2} \mathrm{~s}^{-1}\right)$ and irradiation energy flux $\left(\mu \mathrm{W} \mathrm{cm}{ }^{-2}\right)$ were measured using JAZ Spectrometer (Ocean Optics Inc., Orlando, FL, USA) and used to calculate the relative light intensity expressed as arbitrary units (AU) (Figure 1c).

Berry samples were collected after 6 days from the beginning of the light treatments when berries had reached the stage S4 and started to develop red color on their skin (Figure 1a), and utilized for RNA sequencing and real-time quantitative reverse transcription PCR (qRT-PCR) analyses. For metabolite analyses, light treated S4 stage berries at 6 and 12 days and fully ripe berries after 4 weeks from the beginning of light treatments at stage S5 (Figure 1a) were collected. Approximately 20-25 berries were collected per treatment from three replicate bushes for RNA extraction and metabolite analyses. Immediately after collection, all the berry samples were frozen in liquid nitrogen and stored at $-80^{\circ} \mathrm{C}$ until further used for analyses. 


\subsection{RNA extraction and sequencing}

The frozen berries were ground to a fine powder under liquid nitrogen using mortar and pestle. Total RNA was isolated from approximately $120 \mathrm{mg}$ tissue powder by using Spectrum Plant Total RNA kit (SigmaAldrich, St. Louis, MO, USA) following the manufacturer's instructions. Contaminating DNA was removed with on-column digestion using DNase I (Sigma-Aldrich).

For constructing RNA libraries, the RNA was qualified with both NanoDrop 2000c UV-vis spectrophotometer (NanoDrop Technologies, Wilmington, DE, USA) and Experion Bioanalyzer (Bio-Rad laboratories, Hercules, CA, USA). To segregate the mRNA from total RNA, poly-A was captured using oligo (dT) Dynabeads (Invitrogen, Carlsbad, CA, USA). Before library preparation, all the samples were qualified by an Agilent 2100 Bioanalyzer (Agilent Technologies, Santa Clara, CA, USA). The libraries were prepared with NEBNext Ultra II RNA Library Prep Kit (New England Biolabs Inc., Ipswich, MA, USA). Sequencing of RNA libraries was performed using an Illumina Hiseq2000 platform (Illumina, San Diego, CA, USA) with paired-end sequencing strategy (PE-150bp) at the Novogene sequencing services facility (Cambridge Science Park, UK). Libraries were prepared with three biological replicates for each blue, red, and white (control) light treatments.

\subsection{Transcriptome assembly}

The raw reads from Illumina were initially quality assessed using MultiQC software (Andrews, 2010; Ewels et al., 2016). The adapter contamination was removed using Trimmomatic tool specifically designed for Illumina NGS data (Bolger et al., 2014), followed by the removal of the residual rRNA reads by using sortMeRNA program (Kopylova et al., 2012). The quality checking by MultiQC included assessment of sequence quality score (phred >30), adapter content and position, GC content, and ambiguous bases (Ns). Only the clean filtered reads were used in our downstream analysis. A robust transcriptome was constructed with Trinity v2.9.0 software pipeline (Grabherr et al., 2011) by developing a combined redundant-over assembly from de novo and genome-guided assembly using a bilberry genome sequence of the same bilberry ecotype (Wu et al., 2021, unpublished). The draft genome was indexed and align-mapped to the reads using STAR v2.6.1d software (Dobin et al., 2013). The genome-guided Trinity output was concatenated withde novo transcriptome to form a combined assembly. EvidentialGene tool (Gilbert, 2019) was used to remove the redundancy arising from assemblies. The reads were further mapped to the published highbush blueberry ( $V$. corymbosum cv. Draper v1.0) genome (Colle et al., 2019) using HISAT2 software to improve the annotation of assembly. The best possible coding regions were identified using TransDecoder tool (http://transdecoder.github.io), which identifies a minimal length of open reading frames (ORFs) within reconstructed Trinity transcripts. To assess the completeness of the transcriptome assemblies, BUSCO tool v3.0 (Simão et al., 2015) was used to validate the single copy genes on an evolutionary perspective. Embryophyta orthologous database odb_v.10 (https://busco-archive.ezlab.org/v3/) was used to validate the assembled transcriptomes.

\subsection{Functional annotation of transcriptome}

Functional annotation was performed by using Trinotate pipeline v3.2.1 (http://trinotate.github.io), which utilizes the homology search based on Swissprot, Pfam, and NCBI-BLAST-nr (non-redundant) databases from the CD-hit clustered trinity transcript IDs and TransDecoder derived peptide sequences. The cut-off E-value for the BLAST search was adjusted between $1.0^{-5}$ and $1.0^{-100}$ and the homology search was performed with default parameters. Additional tools, such as SignalP, tmHMM, and RNAMMER (http://www.cbs.dtu.dk/services/), were integrated into the Trinotate pipeline to determine probable signal peptides, transmembrane helices, and residual rRNA transcripts, respectively, in the assembled transcriptome. All the major transcription factor families and transcriptional regulators were determined using the PlantTFcat tool (http://plantgrn.noble.org/PlantTFcat/).

\subsection{Differential gene expression and pathway analysis}

To quantify the gene expression levels from the transcriptomes, we utilized Salmon tool (Patro et al., 2017), which was able to identify and quantify the known gene isoforms. Differentially expressed genes (DEGs) between the light treatments (red vs. control, blue vs. control) were identified using DESeq2 v3.10 software 
package (Love et al., 2014) with false discovery rate (FDR) adjusted p-value set to $0.05 . \log _{2}$ fold change ratio between [?] 2 and [?] -2 was used to obtain the list of up- and down-regulated genes.

Gene Ontology (GO) terms for the transcripts were analyzed using GOseq v3.11 software (https://bioconductor.org/packages/release/bioc/html/goseq.html) in R-package followed by enrichment analysis using Fisher's exact test. For validation and to improve the accuracy in GO determination, the top 500 differentially expressed genes from both the contrasts (red vs. control, blue vs. control) were extracted and annotated with Blast2GO suite (Gotz et al., 2008). KEGG (Kyoto Encyclopedia of Genes and Genomes) pathway enrichment analysis was performed in KOBAS v3.0 tool (Wu et al., 2006) followed by interpreting the KEGG Orthology terms (KO) in KAAS (KEGG Automated Annotation Server) using Vitis viniferaas a reference organism for obtaining the KO identifiers. The original figures and pathway representations were created withwww.biorender.com .

\section{6 qRT-PCR analysis}

Total RNA was isolated from the berry samples using the same method as described above. First-strand cDNA was synthesized using Superscript IV reverse transcriptase (Invitrogen) from $1 \mu \mathrm{g}$ total RNA according to manufacturer's instructions. MJ-MiniOpticon Real-time PCR system (Bio-Rad) was used for qRT-PCR analysis with SsoFast EvaGreen Supermix (Bio-Rad) in 15 4 l volume reaction. The PCR conditions were as follows: initial denaturation at $95^{\circ} \mathrm{C}$ for $30 \mathrm{sec}$ followed by 40 cycles at $95^{\circ} \mathrm{C}$ for $5 \mathrm{sec}$, and $60^{\circ} \mathrm{C}$ for $10 \mathrm{sec}$. Subsequent melting curve analysis, ranging from $65^{\circ} \mathrm{C}$ to $95^{\circ} \mathrm{C}$ with an increment of $0.5^{\circ} \mathrm{C}$ per cycle, was used to assure amplification of only one product. All analyses were performed with three biological replicates and two technical replicates. The results were analyzed using CFX Connect software (Bio-Rad) using 2(-Ct) method, and the relative expression levels were normalized with glyceraldehyde-3-phosphate dehydrogenase (GAPDH ) or Actin. Primer sequences of the genes are listed in Table S1.

\subsection{Analysis of anthocyanins}

Bilberry (S4) samples collected after 6 days of light treatment (equal time-point with transcriptomics samples) were ground and freeze dried in a lyophilizer (Virtis benchtop-K; SP Scientific, Gardiner, NY, USA). Approximately $43 \mathrm{mg}$ dry weight (DW) of lyophilized bilberry powder from each sample was used in extrac-

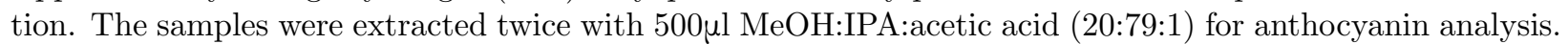
The extracts were evaporated to dryness and resuspended in $100 \mu \mathrm{l} \mathrm{MeOH}$. The extracts were analyzed with UPLC-PDA-Synapt G2 QTOF/HDMS (Waters, Milford, MA, USA) in positive (ESI+) resolution ion mode. Samples were analyzed with capillary voltage at $3.0 \mathrm{kV}$. The source temperature was $120^{\circ} \mathrm{C}$ and desolvation temperature was set to $360^{\circ} \mathrm{C}$, cone gas flow rate was $20 \mathrm{~L} \mathrm{~h}^{-1}$ and desolvation gas flow rate $800 \mathrm{~L} \mathrm{~h}^{-1}$. The compounds were separated on an Acquity UPLC-BEH C18 column $(1.7 \mu \mathrm{m}, 50 \times 2.1 \mathrm{~mm}$, Waters $)$ in $40^{\circ} \mathrm{C}$. The mobile phase consisted of (A) $\mathrm{H}_{2} \mathrm{O}$ and (B) acetonitrile (Chromasolv grade, Sigma-Aldrich, Steinheim, Germany) both containing $0.1 \% \mathrm{HCOOH}$ (Sigma-Aldrich). A gradient of eluents was used as follows: linear gradient of $95 \%$ of A to $5 \%$ in 10 min, then back to $95 \%$ at $10.1 \mathrm{~min}$ and left to equilibrate for $1 \mathrm{~min}$. The injection volume was $2 \mu \mathrm{l}$ and flow-rate of the mobile phase was $0.6 \mathrm{ml} \mathrm{min}{ }^{-1}$. Tray temperature was set to $10^{\circ} \mathrm{C}$. Mass range was set from 100 to 1500 . Peak picking and integration of the peaks was executed with MassLynx V4.2 (Waters) and identification was performed by comparing the exact mass/chemical formula, retention time, UV-spectra and/or previously published data of bilberry secondary metabolites. The relative content of the anthocyanins in arbitrary units (AU) was calculated by normalizing the analyte peaks area with the dry weight of the samples $\left(\mathrm{AU} \mathrm{mg}^{-1} \mathrm{DW}\right)$. Total anthocyanins from $\mathrm{S} 4$ berries after 12 days from light treatment were measured according to Karppinen et al. (2018).

To determine the anthocyanins from S5 stage fully ripe berries, $100 \mathrm{mg}$ of samples were ground to a fine powder and were freeze-dried overnight to remove water content. The samples were further extracted with methanol acidified with $0.1 \% \mathrm{HCl}(\mathrm{v} / \mathrm{v})$ for two hours at room temperature before being centrifuged and the supernatant vacuum spin dried. The pellet was resuspended in $500 \mu \mathrm{l} 20 \%$ methanol and filtered using 0.45 $\mu \mathrm{M}$ PVDF syringe filter (Phenomenex, Torrance, CA, USA). The samples were diluted to 1:10 with 20\% methanol before a $5 \mu \mathrm{L}$ aliquot was injected into a C18 Acclaim Polar Advantage II column $(150 \times 2.1 \mathrm{~mm}$ 
i.d., $3 \mu \mathrm{m}$ particle size; Dionex, Sunnyvale, CA, USA) in a HPLC system (Ultimate 3000; Thermo Fisher Dionex) coupled with a diode array detector (DAD). The column oven temperature was set to $35^{\circ} \mathrm{C}$ and the flow rate was adjusted to $0.350 \mathrm{ml} \mathrm{min}^{-1}$. The mobile phases consisted of $10 \%$ formic acid (A) and a mixture of $45 \%$ methanol, $45 \%$ acetonitrile and $10 \%$ formic acid (B). The gradient was as follows: $100 \%$ A followed by $9 \% \mathrm{~B}$ in $\mathrm{A}$ for $0-12 \mathrm{~min}, 35 \% \mathrm{~B}$ in $\mathrm{A}$ for $12-25 \mathrm{~min}, 50 \% \mathrm{~B}$ in $\mathrm{A}$ for $25 \mathrm{~min}$, and $9 \% \mathrm{~B}$ in A for 30-35 min. The identified anthocyanin peaks were compared with that of known authentic standards and monitored at $254 \mathrm{~nm}, 280 \mathrm{~nm}, 320 \mathrm{~nm}$, and $520 \mathrm{~nm}$. The samples were quantified using a calibration curve and expressed as cyanidin 3- $O$-galactoside equivalents. All analyses were performed with three biological replicates.

\subsection{Statistical analysis}

The statistical analysis of LC-MS profiling data was performed with MetaboAnalyst 5.0 tool (Chong et al., 2019). Differences in anthocyanin levels among the light treatments were analyzed by one-way ANOVA followed by Turkey's post-hoc test. Statistically significant differences between the light treatments analyzed by qRT-PCR were determined by independent samples $t$ - test in SPSS Statistics program v26 (IBM corporation, New York, NY, USA). Pearson's correlation matrices were used to visualize the statistical relationship from correlation coefficients between selected gene expression levels and dominant anthocyanin concentrations (delphinidin 3-galactoside and delphinidin 3-arabinoside) with p-value set to $<0.05$. ANOVA and Pearson's correlation analysis were performed using Origin Pro software v2020b (Origin Lab Corporation, Northampton, MA, USA).

\section{Results}

\subsection{Bilberry transcriptome sequencing and functional annotation}

The raw sequencing reads of the bilberry transcriptomes yielded approximately $56 \mathrm{~Gb}$ of data and reached approximately $8 \mathrm{~Gb}$ per sample data. The read number for control samples was 70,627,656 bases, while it was $82,253,205$ bases for the red light treated samples and 78,192,227 bases for the blue light treated samples (Table S2). MultiQC analysis proved that the processed reads were of good quality with Phred score $>36$ (Table S2). Read-mapping to a recently published $V$. corymbosum genome (Colle et al., 2019) resulted in $75-77 \%$ of total reads mapped including $\sim 50 \%$ uniquely mapped to the genome. Using a draft genome of bilberry (Wu et al., 2021, unpublished), representing the same bilberry ecotype as our samples, enabled unique mapping of $83.5 \%$ of the filtered reads. A total of 671,952 transcripts and 472,876 unigenes were generated from the combined transcriptome assembly with mean contig lengths of $911 \mathrm{bp}$ and $720 \mathrm{bp}$, respectively (Table 1). BUSCO analysis revealed that the combined assembly had $97.4 \%$ complete sequences when searched within 1375 orthologous groups of embryophyta_odb9 lineage (Table S3). The scores were slightly improved compared to genome-guided assembly indicating that the combined transcriptome in our analysis is a robust assembly and was subsequently used in this study.

In total, around 25,316 (61\%) of putative protein IDs of bilberry transcripts showed significant hits in Swissprot and $25,280(61 \%)$ in Pfam databases (Figure 2a). Around $60 \%$ of the sequences had hits with eggNOG (clusters of orthologous groups) and relatively high number of hits (65\%) obtained from the KEGG database (Figure 2a). BLAST hits distribution among the top-25 species showed the highest homology in Rhododendron williamsianum (33\%) followed by Camelia sinensis var. (25\%) and Actinidia chinensis var. $(16 \%)$ (Figure S1). The top-hit species with some considerable matches obtained from the top DEGs $(0.2 \%$ $2.6 \%$ ) showed sequence similarities with $V$. myrtillus, V. macrocarpon and V. corymbosum .

There were 49,105 commonly co-expressed genes detected among the transcriptomes of the three different light treatments (Figure 2b). The distribution between the different light treatments is visualized in a Venn diagram showing that 1816 and 1686 genes were uniquely expressed among the red and blue light treatments, respectively (Figure $2 \mathrm{~b}$ ). The BLAST sequence similarity distribution within the query sequences (E-value cut-off $1.0^{-5}$ ) showed high number of positives to that of aligned reads length in the range of $70-90 \%$ suggesting a strong match between query and assembled known sequences from databases (Figure S2).

3.2 Differential expression analysis between light treatments and enrichment (GO, KEGG) 


\section{analysis}

The gene expression levels quantified from the read counts generated from the transcriptome showed 14,105 differentially expressed genes (DEGs). The FPKM counts (fragments per kilobase of transcript per Million mapped reads) were aggregated from three replicates of each light treatment (Figures S3, S4). Hierarchical clustering analysis showed clear differences in the expression patterns between the light treatments (Figure 3a). In red light treated berries, high number of DEGs corresponding to 7327 up-regulated genes and 1545 down-regulated genes (Figure 3b) were detected when compared with blue light treatment yielding 3686 up-regulated and 1547 down-regulated genes (Figure 3c) as visualized using volcano plots.

GO Enrichment analysis classified the DEGs according to their functions and properties into three major categories Biological process (BP), Molecular function (MF) and Cellular process (CC) (Figure 4a). An average of $60-65 \%$ of unigenes were assigned GO terms either through one of the homology searches from Pfam, BLASTx and BLASTp databases. The top significantly enriched GO terms across these three categories from our two comparison subsets showed that in CC 'intracellular' and 'cellular anatomical entity' were the top sub-categories (Figure 4a). In the MF category 'catalytic activity', 'binding' and 'transporter' activities were found to be abundant (Figure 4a). Both these categories have similar number of GO terms assigned from the sequences but with two contrasting results. In the BP category, the sequences assigned to 'metabolic' and 'cellular' process were relatively higher in red light than blue light treatment. The GO terms assigned to 'localization', 'signaling' and 'response to stimulus' sub-categories were contrasting between the light treatments (Figure 4a). Some of the unigenes were also classified in 'rhythmic processes' and 'pigmentation' in the BP category. Hence, we further investigated the biological process (BP) category by direct count of sequence distribution among the top DEGs. GO terms, such as "oxidation-reduction process", "protein phosphorylation", and "regulation of transcription", were the top ones with relatively higher number of assigned sequences found in red light treated samples than blue light treatment. Some annotated sequences related to sugar metabolism, shikimate, chorismate, lignin, cutin and sterol biosynthetic process were also determined with additional GO terms assigned to anthocyanin containing compounds and flavonoid biosynthetic process were obtained from red light treatment (Figure 4b). The KEGG pathways significantly enriched by adjusting FDR corrected/p-value to $<0.05$ using Benjamini-Hochberg method showed that a higher number of gene ratio fell on metabolic pathways and secondary metabolite biosynthesis predominantly the phenylpropanoid biosynthesis followed by terpenoid biosynthesis with considerable number of gene counts (Figure 4c, d). In addition, the red light treatment has also significantly enriched the fatty acid, galactose, starch and sucrose metabolism related pathways (Tables S4, S5, S6, S7). All the DEGs and corresponding unigene IDs analyzed throughout this study is provided in Table S8.

\subsection{Identification of transcription factor (TF) families}

Blast2GO suite identified Gene Ontology (GO) terms and protein domain signatures from top 500 DEGs utilizing InterproScan (IPS) database. The analysis indicated that the top category was a protein kinase domain followed by $M Y B / S A N T$ domain, $A P 2 / E R F, N B-A R C$, and $b H L H$ domains (Figure S5). A total of 976 unigenes were identified as putative TFs and regulators from the transcriptome categorized into 72 transcription factor families, which are likely to have a role in red and blue light-mediated transcriptional regulation in various metabolic pathways. Among them, C2H2 was the most prominent TF family category with 117 unigenes followed by $C C H C(Z n)$ (99 unigenes), $R R$ - $A$ type (42 unigenes), $M Y B-H B$ like (62 unigenes),,$H H L H$ (44 unigenes) and $W D-40$ like transcription factors (40 unigenes) (Figure S6). Some of the earlier identified TFs known to be involved in bilberry ripening (Nguyen et al., 2018), such as NAM (NAC family TFs) with 13 unigenes, WRKY TFs with 17 unigenes and MADS -like TFs with 13 unigenes, were identified as differentially expressed in response towards red and blue light treatment. Another class of TFs called AP2-EREBP (APETALA2/EF) belonging to ethylene hormone responsive transcription factor family, was also significantly expressed among the DEGs (38 unigenes).

\subsection{DEGs involved in red and blue light signaling}

The unigenes corresponding to phytochrome B (PhyB ) were found to be highly up-regulated in red light 
treatment. Whereas, cryptochrome (Cry2) transcripts were found in high levels under blue light (Figure 5). COP1 expression level was found to be higher in red light treatment with blue light down-regulating its expression. Both the light treatments showed similar trends of COP1 and HY5expression. "K2a and PRR5, which are key regulators in plant circadian rhythms, did not vary markedly in their expression levels between the red and blue light treatments. Flowering locus gene-T $(F T)$ involved in photoperiodism was highly up-regulated $(2,8 \log 2 \mathrm{FC})$ upon blue light treatment (Figure 5$)$.

\subsection{DEGs from anthocyanin biosynthesis and its associated regulatory complex}

All the major identified unigenes involved in the phenylpropanoid pathway, such as $P A L$, cinnamate 4hydroxylase $\left(\mathrm{C}_{4} \mathrm{H}\right)$ and 4-coumarate $\mathrm{CoA}$ ligase $(4 \mathrm{CL})$, were up-regulated upon both red and blue light treatments (Figure 6). Caffeoyl CoA $O$-methyltransferase ( $c C o A o M T$ ) expression was found to be at a relatively higher level in blue light compared to red light treatment also with similar expression levels identified in peroxidases and cationic peroxidases (Prx , cPrx) genes leading to lignin biosynthesis via the $p$ -coumaryl alcohol branch pathways (Figure 6).

All the anthocyanin biosynthetic pathway structural genes were up-regulated both in red and blue light (Figure 6). The key biosynthetic enzyme gene $C H S$ was found with a log2 fold increase of 3,3 and 3 between red and blue treatment, respectively. Expression of $C H I$ and $F 3 H$ was found to be at a higher level in red light treatment when compared with the blue light treatment. The changes in expression levels of $F 3$ ' $H$, $D F R$ and $A N S$ genes were found to be slightly higher in blue light treatment whereas the key gene involved in the delphinidin branch pathway, $F 3^{\prime}{ }^{\prime}{ }^{\prime} \mathrm{H}$, and the specific gene for anthocyanin biosynthesis, UFGT , were found to be highly up-regulated in the response towards red light (Figure 6).

R2R3 MYBs (8 unigenes) and bHLHs (6 unigenes) were found among the top 500 DEGs. All the identified TFs of MBW complex from top DEGs and corresponding gene ID with IPS domains are provided in the Table S9. SQUAMOSA-MADS box (TDR-type) TFs, such as AGAMOUS(AGL 80, 21,15, 61,62) and MADS $(2,3)$, were significantly up-regulated in red light treatment and down-regulated under blue light treatment (Figure 6). In contrast, blue light up-regulated DEF2and PHE2 type MADS box transcription factors and red light down-regulated SPL4 type TFs (Figure 6), which are all linked to regulating circadian rhythm and flowering. The expression level of $M Y B A 1$, the key regulatory gene of anthocyanin biosynthesis, was upregulated by both red and blue light treatments, whereas $M Y B P A 1.1$ was found comparatively higher level in red light treatment. Most of the eight R2R3-MYB genes categorized as DEGs expressed very similarly in both the light treatments except MYBPA1.1, MYBPA3 and MYBC2 which were found slightly lower levels in blue light treatment (Figure 6). All the bHLH TFs categorized under DEGs (bHLH6 (MYC2), bHLH130, bHLH137, bHLH147, bHLH68 andbHLH79) were found up-regulated in both light treatments and expressed in similar levels.

\subsection{DEGs from carotenoid and ABA metabolism}

The carotenoid pathway genes, such as phytoene synthase $(P S Y), \beta$-carotene hydroxylase $(B C H)$, and carotenoid $\beta$-ring hydroxylase of cytochrome $\mathrm{P} 450$ family $(C Y P 450-B C H)$, were up-regulated in both red and blue light treatments with the exception of $\zeta$-carotene desaturase (ZDS) and lutein deficient 1-like(Lut1) with lower expression levels in blue light treatment (Figure 6). In addition, the important carotenoid cleavage gene $N C E D$, the key cleavage gene in abscisic acid biosynthesis, was up-regulated in both the light treatments but higher in red light treatment (Figure 6). On the other hand, $A B A-8^{\prime}$ hydroxylase, which is the first step in the ABA catabolism route was highly up-regulated only under red light treatment but down-regulated (to -1.88-fold change) in blue light treatment. There were three unigenes identified as ABA-receptors pyrabactin resistance like gene $(P Y L-4,5,8)$ of which two of them ( $P Y L$ 5,8) were down-regulated to 2-3 folds lower in response to blue light (Figure $6 \mathrm{~b}$ ). ABA insensitive ( $A B I 5$ ) $\mathrm{TF}$ was found in similar levels in both the light treatments.

The related gene expression obtained from qRT-PCR analyses for all the key anthocyanin and ABA biosynthetic genes discussed above (CHS, F3' $\left.H, F 3^{\prime} 5^{\prime} H, D F R, A N S, U F G T, M Y B A 1, N C E D\right)$ and identified unigenes from RNA-seq dataset were correlated which showed a higher correlation $\mathrm{R}^{2}$ values of 0.9 with red 
vs control and 0.8 with blue vs control contrasts (Figure S7).

\subsection{DEGs involved in vesicular trafficking}

DEGs identified and annotated as group of genes related to SNARE-domain family of transporter proteins, such as Stx 5,6,7(syntaxin), Sec 20,22 and Ykt6, were highly up-regulated in response towards the red light treatment (Figure 7). The syntaxin genes (Stx1-4), usually found in apical plasma membrane, were up-regulated in red light and down-regulated in blue light treatment. We also identified two unigenes from DEGs annotated as $A B C$ family of transporter proteins (ATP-binding cassette sub-family B), which showed same expression trend to syntaxin genes suggesting the involvement of ABCs in vesicular transport, possibly together with SNARE's (Figure 7).

\subsection{Elevated anthocyanin content under red and blue supplemental light}

Metabolite profiling with UPLC-HDMS was performed from light treated berry samples after 6 days (same time point for transcriptome libraries). The red, blue and control samples from the metabolite analysis were separated in the first component in the Principal Component Analysis (PCA), explaining 32.3\% of the variation (Figure S8). The heat map analysis of metabolite profiling data shows large number of significantly different metabolites (407 metabolites of total 700, ANOVA, p<0.05); anthocyanins localized in the middle part of the clusters (Figure S9). The results showed consistent and significant increase in all the anthocyanin compounds under both the red and blue light treatments when compared with control (Figure 8a). Delphinidin galactosides/glucosides were detected 11-fold higher in red light when compared to other light treatments followed by significant increase in all the cyanidin, petunidin and malvidin glycosides (Figure 8a). Also, the total anthocyanins quantified in ripening berries (S4) after 12 days of light treatment showed higher amounts of anthocyanin accumulation in red light followed by blue light treatments compared with ambient white light control (Figure 8b).

Similar trend was also observed in S5 stage fully ripe berries although the difference was not as apparent (Table S10). Quantitative analysis with HPLC confirmed that the two major delphinidin glycosides, delphinidin-3-galactoside and delphinidin 3-arabinoside, were found at significantly higher amounts in red

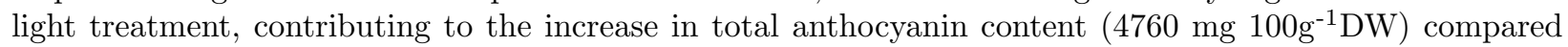
to that of blue light and the control samples. The trend is followed by the increase in levels of cyanidin and petunidin glycosides in red light treated berries. A very low amount of peonidin-3-glucoside was detected in all the samples which was not generally influenced by different light treatments (Table S10).

Pearson's correlation matrices between the dominantly found anthocyanins quantified using HPLC (delphinidin 3-galactoside and delphinidin 3-arabinoside) and the transcript levels related to the flavonoid, carotenoid, ABA pathway genes, and ABA-receptors from both light treatments (Figure 9) indicated strong links between ABA and anthocyanin biosynthesis especially with red light. We also found statistically significant positive correlations among gene-metabolite interactions for red light compared to blue light treatment.

\section{Discussion}

It has been shown in many fruits that specific light spectral wavelengths can influence the biosynthesis of anthocyanins and other polyphenolic compounds (Jeong et al., 2004; Kokalj et al., 2019; Koyama et al., 2012; Tao et al., 2018). However, the molecular mechanisms underlying the light quality regulated fruit anthocyanin biosynthesis are not well understood. In this study, we used a comparative transcriptomics approach to analyze the effect of light quality on anthocyanin biosynthesis in ripening fruit of bilberry producing anthocyanins from both cyanidin and delphinidin branches. The light treatments were given to unripe green (S3) bilberry fruits, as this stage has been shown earlier to be reactive towards changes in spectral light wavelengths affecting anthocyanin biosynthesis (Zoratti et al., 2014b). Here, we showed that the anthocyanin concentration and profile in ripening berries was affected especially by red light but also

blue light treatment, and our results indicate differences in light signaling pathways between red and blue light in regulation of anthocyanin biosynthesis.

Light quality modulates light signal perception and transduction 
In natural conditions, plants encounter varying light spectral conditions. For example, in the latitudes close to Arctic circle, the radiation flux from a distinct solar spectrum (UV-A/B ratio, blue and red/far-red wavelengths) changes compared to southern latitudes and this has been shown to favor higher accumulation of flavonoids in northern vegetation, including wild bilberries (Jaakola \& Hohtola 2010; Zoratti et al., 2014a). Delphinidins are the major class of constituting anthocyanins found abundant in northern clones compared with higher cyanidin proportions found in southern clones (Zoratti et al., 2016). Also, in forests the top canopy absorbs most of the essential red and blue wavelengths and only the green and far-red wavelengths are reflected by foliage to lower parts of the plant (Holopainen et al., 2018). In bilberry, populations grown under direct sunlight have demonstrated increased bioactive compounds and bioavailability compared with plants growing under forest canopy (Eckerter et al., 2019). However, the even distribution through the foliage can be achieved by modern energy efficient LEDs because the irradiation maxima from these supplemental lightings are often higher than plant's absorption peaks. Our study showed that during the red light LED treatment, PhyBexpression was upregulated together with COP1 and HY5 , the key genes involved in photomorphogenesis, alongside photoperiodism-related early flowering (ELF3 ) and "K2agenes (Figure 5). On the other hand, the blue light up-regulated cryptochrome (CRY2) but down-regulated COP1 and HY5in tandem as its photoreactive mechanism which might reveal an early photomorphogenesis occurred upon light treatment as a spontaneous response. It is also to be noted that bHLH6 (MYC2), which is a negative regulator of blue light induced photomorphogenesis and was positively expressed (Yadav et al., 2005), might have played a crucial role in blue light signaling mechanism.

\section{Supplemental red and blue light irradiation positively affects anthocyanin biosynthesis and accumulation}

Red and blue wavelengths have optimal chlorophyll absorption and photosynthetic efficiency (Massa et al., 2008), and both appear to be effective in promoting anthocyanin biosynthesis in various horticultural crops (Bian et al., 2014; Zhang et al., 2018). Our results showed that both the light treatments up-regulated all the anthocyanin biosynthetic genes in bilberry fruit, including all the bottleneck flavonoid biosynthetic genes CHS, F3H and ANS described in Vaccinium fruits (Primetta et al., 2015; Zorenc et al., 2017; Günther et al., 2020). This led to the higher accumulation of anthocyanins under both red and blue light treatments compared to control fruits. Furthermore, the expression level of UFGT, the last gene in the anthocyanin pathway, and $F 3^{\prime} 5^{\prime} H$, the gene directing dihydroflavonol precursors to delphinidin biosynthesis, were found to be highly up-regulated under the red light treatment. This changed the anthocyanin profile towards delphinidin glycosides in fully ripe berries under red light. Our results suggest that UFGT and F3'5' $H$ are separately regulated and highly responsive to red light in ripening bilberry fruit. In climacteric fruits, studies have shown blue light to influence anthocyanin biosynthesis as shown in pear (Tao et al., 2018). Whereas, in comparison with non-climacteric fruits, a recent study in strawberry showed that both red and blue light were able to increase gene expression levels of flavonoid biosynthetic pathway with red light found to be slightly more effective in inducing anthocyanin accumulation (Zhang et al., 2018).

Anthocyanin biosynthesis is directly regulated by the MBW complex andR2R3 $M Y B$ expression and to some extend $b H L H$ expression. A high number of DEGs representing $M Y B$ and $b H L H$ genes was found from our RNA-Seq libraries indicating that their regulation is strongly influenced by the light spectral quality. $M Y B A$ - type TFs have been identified as the key regulators activating anthocyanin biosynthesis in fruits including Vaccinium berries (Plunkett et al., 2018; Die et al., 2020). Also, MYBPA1 has been suggested to have a regulatory role in anthocyanin biosynthesis based on our previous studies (Primetta et al. 2015; Günther et al., 2020; Karppinen et al., 2021 unpublished). In this study, both MYBA1 and MYBPA1 were up-regulated by red and blue light. Especially $M Y B P A 1$ was induced by red light, which has been recently suggested as one of the key genes in regulating delphinidin branch during bilberry ripening (Karppinen et al., 2021 unpublished). Other bilberry MYB sequences homologous to related MYBPA2, MYB5 and MYBC2 genes were also found among top 500 DEGs which are likely to be involved in regulatory mechanisms during ripening and flavonoid biosynthesis. Some of the bHLH TF sequences which were up-regulated in both red and blue light treatments, such as $b H L H 79$ have been previously reported in grapes towards light induced anthocyanin biosynthesis (Ma et al., 2019). Also, bHLH6 (MYC2) transcription factor is reported to be 
commonly involved in light and ABA signaling pathways (Yadav et al., 2005).

\section{Differences in carotenoid biosynthesis and ABA metabolism suggest red light regulation through ABA}

A significant increase in the expression of the carotenoid early biosynthetic genes and late cleavage genes has earlier been found in bilberry fruit ripening under red light (Karppinen et al., 2016b). Our results are in agreement with this study but also showed that not only the red light but also blue light treatment up-regulated expression levels of many of the carotenoid biosynthetic genes. However, the expression of $Z D S$ and $C Y P 450-B C H$ genes branching towards $\alpha$-carotene and further to lutein biosynthesis (LUT1) varied between the red and blue light treatments (Figure 6). Earlier it has been shown that the up-regulated expression levels of carotenoid biosynthetic genes in red light conditions led to low levels of carotenoids indicating elevated cleavage reactions (Karppinen et al., 2016b).

The cleavage of carotenoids leads to the production of plant hormone ABA through action of the key enzyme NCED (Karppinen et al., 2013). Many studies have demonstrated the exogenous application of ABA increasing the anthocyanin levels of non-climacteric fruits when applied at the time of fruit ripening (Wheeler et al., 2009; Ferrero et al., 2018; Karppinen et al., 2018). In strawberry, the effect of endogenous ABA levels in response to light-regulated anthocyanin biosynthesis has been reported through the activation of FvMYB10, the key gene in strawberry anthocyanin biosynthesis (Kadomura-Ishikawa et al., 2015). In our study, the red light treatment induced higher expression of NCED compared to control and blue light treatment. Also, we showed that expression levels of $A B A-8^{\prime}$ hydroxylase gene, responsible for degradation of ABA, increased 2-fold higher in red light treatment and was down-regulated in blue light treatment. It has been shown that the endogenous levels of ABA in plant cells are maintained only by the inhibition of this enzyme (Kondo et al., 2012). A similar trend of increase in expression of NCED and ABA-8' hydroxylase genes alongside higher anthocyanin accumulation in response to red light irradiation was observed earlier in grapevine (Kondo et al., 2014). Rodyoung et al. (2016) have shown that in grapes, the expression of both of these genes was higher at veraison in response to red and blue light irradiation. In many fruits, the ABA catabolism might not directly coincide with anthocyanin biosynthesis but the upstream ABA biosynthesis and endogenous levels of ABA may be directly involved in interaction with the flavonoid pathway. Here we show that in ripening bilberry fruit the red light activates both the biosynthetic and catabolic ABA pathways and correlates with anthocyanin accumulation. Furthermore, the changes in delphinidin levels showing a strong correlation with anthocyanin biosynthesis and direct regulatory genes, ABA signaling and metabolism genes (Figure 9) suggests a well-orchestrated regulatory network of ABA-regulated anthocyanin biosynthesis occurring under supplemental red light.

The signal transduction from ABA to the regulation of anthocyanin biosynthesis has been reported earlier in other fruit species, such asLycium fruits, where ABA was found to interact directly with the MBW complex and other key flavonoid genes (Li et al., 2019). In our study, we showed that light treatments during ripening process activated signal transduction cascade via ABA signaling lead to anthocyanin accumulation. It has been shown in one of the recent studies that red light increased the expression level of AGAMOUS -like (AGL) regulators in tomato (Solanum lycopersicum L.) during fruit ripening (Zhang et al., 2020). These AGL-like MADS box TFs are also orthologs to VmTDR4 in bilberry, a key player in bilberry anthocyanin accumulation (Jaakola et al., 2010). This provides us with an understanding that red light might mediate ABA-regulated anthocyanin biosynthesis through SQUAMOSA-MADS box type TF's, abscisic acid binding receptors such as pyrabactin resistance like $(P Y R / P Y L), \mathrm{ABA}$ insensitive $(A B I 5)$ gene and MBW complex TFs (Chung et al., 2019). It should also be noted that the $S P L$ type of TFs (SQUAMOSA promoter binding like) that were up-regulated in response to blue light can be associated with the increase in expression of FLOWERING LOCUS-T (FT) gene involved in flowering and circadian clock related mechanisms as the TF acts downstream to FT expression (Wang et al., 2009).

\section{Supplemental light triggers vesicular trafficking in ripening berry accumulating anthocyanins}

The transportation of anthocyanins into vacuoles is usually trafficked intra-cellularly by different classes of 
transporter proteins, such as MATEs, ABCs, and GSTs (Petrussa et al., 2013). The transporters involved in fruit anthocyanin transport in relation to light quality response have not been studied. However, our results demonstrated that a set of genes, including Stx, Bos1, Gos1, Ykt6, Sec20, Sec 22, andSyp7 were highly up-regulated in response to red light treatment. These genes belonged to the SNARE-domain protein family, which includes the common syntaxin-like (Stx, Syp ) genes. Interestingly, the unigenes annotated as Stx -like $(1,4)$ type along with an $\mathrm{ABC}$ transporter were up-regulated in response to red light treatment and reacted in the opposite manner to that of blue light treatment. Hence, SNARE domain transporters are candidates to be involved in vesicular trafficking of anthocyanins or trans-membrane transport from endoplasmic reticulum (ER) to golgi and endosomes. It also indicates the potential role of syntaxin genes differentially responding to light-induced anthocyanin transport mechanisms. These different types of SNAREs, barring the ER localized Syp and Sec are usually localized in endosomes and in trans-golgi network (Kim et al., 2012). Our results may indicate a new route for anthocyanin sequestration and transportation in fruit tissues. The proposed vesicular trafficking model of anthocyanins in fruits might be also co-regulated by otherGST s and $A B C$ transporters in addition to the SNARE-domain type proteins before depositing as anthocyanic vacuolar inclusions (AVI) in the vacuole (Zhao, 2015). This mechanism could also relate to the plant tissues that accumulate higher anthocyanin levels under high intensity light and needs to be investigated further.

\section{Conclusion}

Our RNA-seq analyses from the time of fruit ripening show that both red and blue wavelength are capable of inducing a high number of up-regulated genes and metabolic pathways, including flavonoid, phenylpropanoid, carotenoid, terpenoid backbone biosynthetic pathways and sugar metabolism. Blue and especially red light were effective in inducing anthocyanin and delphinidin accumulation but through different signal transduction routes. The blue light triggered early photomorphogenesis via $C R Y 2 / C O P 1$ interaction that potentially combined with positive regulators, such as MYBA and $H Y 5$ to induce the expression of anthocyanin biosynthetic genes during onset of ripening. Red light treatment instead positively up-regulatedPhyB and all the major flavonoid genes, including the anthocyanin (UFGT) and delphinidin $\left(F 3^{\prime} 5^{\prime} H\right)$ routes, key ABA biosynthetic gene (NCED) and ABA degrading $A B A-8$ 'hydroxylase genes. Our results provide an insight into the role of endogenous $\mathrm{ABA}$ accumulation and degradation as positive signaling factors leading to increased levels of anthocyanin accumulation via the ABA-signal transduction mechanism during the ripening process under red-light. We also found the expression of SNARE complex-related vesicle trafficking genes to be highly expressed in red light treated berries, which might provide clues into the possible sequestration and transport mechanisms via endosomes in tissues with higher anthocyanin accumulation, but need further investigation. Our high-quality transcriptome dataset will be a useful genomics resource in future bilberry research and Vaccinium breeding programs.

\section{Acknowledgments}

The authors would like to thank Leidulf Lund for the help in setting up light experiments at the Phytotron facility at UiT The Arctic University of Norway. We are grateful for the UiT-BFE faculty's mobility grant to AS allowing the visit to Plant and Food Research, New Zealand. The research mobility was supported by the New Zealand Ministry for Business, Innovation, and Employment (MBIE) Endeavour programme 'Filling the Void: boosting the nutritional content of NZ fruit' (contract C11X1704). The work was also financially supported by NordPlant (NordForsk grant no. 84597).

\section{Electronic data availability}

The bilberry RNA-seq raw reads data files have been deposited in NCBI's SRA database and made available under the project number PRJNA-

\section{(URL-}

\section{Author contributions}

LJ conceptualized the project and along with KK, IM and AS designed the experiment. AS performed the experiments, analyzed the data, and wrote the manuscript. DJ contributed in RNA-seq bioinformatics 
pipeline and analysis. APD contributed with the HPLC analysis of anthocyanins. NS performed metabolic profiling with LC-MS. LJ, KK, IM and RVE contributed in the editing and proofreading of the manuscript draft. All authors have read and approved the manuscript.

\section{Conflict of interest}

The authors declare that they have no conflict of interest.

\section{References}

Andrews, S. (2010). FastQC: A quality control tool for high throughput sequence data [online]. Available online at: http://www.bioinformatics.babraham.ac.uk/projects/fastqc/ Ballaré, C. L. (2014). Light regulation of plant defense. Annual Review of Plant Biology, 65, 335-363. doi: https://doi.org/10.1146/annurev-arplant050213-040145 Behrens, C. E., Smith, K. E., Iancu, C. V., Choe, J.Y \& Dean, J. V. (2019). Transport of anthocyanins and other flavonoids by the arabidopsis ATP-binding cassette transporter AtABCC2. Scientific Reports, 9:1-15. doi:https://doi.org/10.1038/s41598-018-37504-8Bhatnagar, A., Singh, S., Khurana, J. P., \& Burman, N. (2020). HY5-COP1: the central module of light signaling pathway. Journal of Plant Biochemistry and Biotechnology, 29(4), 590-610. doi: https://doi.org/10.1007/s13562-020-00623-3 Bian, Z., Yang, Q. \& Liu, W., (2014). Effects of light quality on the accumulation of phytochemicals in vegetables produced in controlled environments: a review. Journal of the Science of Food and Agriculture, 95(5), 869-877. Bolger, A., Lohse, M., \& Usadel, B. (2014). Trimmomatic: a flexible trimmer for Illumina sequence data. Bioinformatics, 30(15), 2114-2120. doi:https://doi.org/10.1093/bioinformatics/btu170Briggs, W. R., \& Olney, M. A. (2001). Photoreceptors in plant photomorphogenesis to date. Five phytochromes, two cryptochromes, one phototropin, and one superchrome. Plant Physiology, 125, 85-88. doi: https://doi.org/10.1104/pp.125.1.85 Bujor, O., Le Bourvellec, C., Volf, I., Popa, V. \& Dufour, C. (2016). Seasonal variations of the phenolic constituents in bilberry (Vaccinium myrtillus L.) leaves, stems and fruits, and their antioxidant activity. Food Chemistry, 213,58-68. Chanoca, A., Kovinich, N., Burkel, B., Stecha, S., Bohorquez-Restrepo, A., Ueda, T., \& Otegui, M. S. (2015). Anthocyanin vacuolar inclusions form by a microautophagy mechanism. Plant Cell, 27(9), 2545-2599. doi: https://doi.org/10.1105/tpc.15.00589Chen, M., Chory, J., \& Fankhauser, C. (2004). Light signal transduction in higher plants. Annual Review of Genetics, 38(1), 87-117. doi:https://doi.org/10.1146/annurev.genet.38.072902.092259Cherian, S., Figueroa, C. R., \& Nair, H. (2014). 'Movers and shakers' in the regulation of fruit ripening: a cross-dissection of climacteric versus non-climacteric fruit. Journal of Experimental Botany. Bot. 65, 4705-4722. doi: https://doi.org/10.1093/jxb/eru280 Chong, J., Wishart, D. S., \& Xia, J. (2019). Using metaboanalyst 4.0 for comprehensive and integrative metabolomics data analysis. Current Protocols in Bioinformatics, 68, e86. doi:https://doi.org/10.1002/cpbi.86Chu, W. K., Cheung, S. C., Lau, R. A., \& Benzie, I. F. (2011). Bilberry (Vaccinium myrtillus L.). Herbal Medicine, 20115386, 55-71. Chung, S. W., Yu, D. J., Oh, H. D., Ahn, J. H., Huh, J. H., \& Lee, H. J. (2019). Transcriptional regulation of abscisic acid biosynthesis and signal transduction, and anthocyanin biosynthesis in 'Bluecrop' highbush blueberry fruit during ripening. Plos One, 14(7), e0220015. Colle, M., Leisner, C., Wai, C., Ou, S., Bird, K., \& Wang, J. et al. (2019). Haplotype-phased genome and evolution of phytonutrient pathways of tetraploid blueberry. Gigascience, 8(3), giz012. doi: https://doi.org/10.1093/gigascience/giz012 Die J.V., Jones R.W., Ogde E.L., Ehlenfeldt M.K., Rowland L.J. (2020). Characterization and analysis of anthocyanin-related genes in wild-type blueberry and the pink-fruited mutant cultivar 'Pink Lemonade': new insight into anthocyanin biosynthesis. Agronomy 10, 1296. Dobin, A., Davis, C., Schlesinger, F., Drenkow, J., Zaleski, C., \& Jha, S., Batut, P., Chaisson, M., \& Gingeras, T.R., (2013). STAR: ultrafast universal RNA-seq aligner. Bioinformatics, 29(1), 15-21.doi: https://doi.org/10.1093/bioinformatics/bts635Eckerter, T., Buse, J., Forschler, M., \& Pufal, G. (2019). Additive positive effects of canopy openness on European bilberry (Vaccinium myrtillus) fruit quantity and quality. Forest Ecology and Management, 433, 122130. doi:https://doi.org/10.1016/j.foreco.2018.10.059Ewels, P., Magnusson, M., Lundin, S., \& Kaller, M. (2016). MultiQC: summarize analysis results for multiple tools and samples in a single report. Bioinformatics, 32(19), 3047-3048. doi:https://doi.org/10.1093/bioinformatics/btw354Feller, A., MacHemer, K., Braun, E. L., \& Grotewold, E. (2011). Evolutionary and comparative analysis of MYB and bHLH plant 
transcription factors. Plant Journal, 66 (1), 94-116. doi: https://doi.org/10.1111/j.1365-313X.2010.04459.x Ferrara, G., Mazzeo, A., Matarrese, A. M. S., Pacucci, C., Punzi, R., Faccia, M., Trani, A., \& Gambacorta, G. (2015). Application of abscisic acid (S-ABA) and sucrose to improve colour, anthocyanin content and antioxidant activity of cv. Crimson seedless grape berries.Australian Journal of Grape and Wine Research, 21(1), 18-29. doi: https://doi.org/10.1111/ajgw.12112Ferrero, M., Pagliarani, C., Novak, O., Ferrandino, A., Cardinale, F., Visentin, I., \& Schubert, A. (2018). Exogenous strigolactone interacts with abscisic acid-mediated accumulation of anthocyanins in grapevine berries. Journal of Experimental Botany, 69(9), 2391-2402. doi: https://doi.org/10.1093/jxb/ery033Gilbert, D. (2019). Longest protein, longest transcript or most expression, for accurate gene reconstruction of transcriptomes?bioRxiv, 829184 doi: https://doi.org/10.1101/829184Gotz, S., Garcia-Gomez, J., Terol, J., Williams, T., Nagaraj, S.H., \& Nueda, M. J., Robles, M., Talon, M., Dopazo, J., \& Conesa, A. (2008). High-throughput functional annotation and data mining with the Blast2GO suite. Nucleic Acids Research, 36(10), 3420-3435. doi:https://doi.org/10.1093/nar/gkn176Grabherr, M., Haas, B., Yassour, M., Levin, J., Thompson, D., \& Amit, I. et al. (2011). Full-length transcriptome assembly from RNA-Seq data without a reference genome. Nature Biotechnology, 29(7), 644-652. doi: https://doi.org/10.1038/nbt.1883 Gunther, C. S., Dare, A. P., McGhie, T. K., Deng, C., Lafferty, D. J., Plunkett, B. J., Grierson, E., Turner, J. L., Jaakola, L., Albert, N. W., \& Espley, R. V. (2020). Spatiotemporal modulation of flavonoid metabolism in blueberries. Frontiers in plant science, 11, 545. doi: https://doi.org/10.3389/fpls.2020.00545Heysieattalab, S., \& Sadeghi, L. (2020). Effects of delphinidin on pathophysiological signs of nucleus basalis of meynert lesioned rats as animal model of alzheimer disease. Neurochemical Research, 45(7), 1636-1646. doi:https://doi.org/10.1007/s11064020-03027-wHolopainen, J., Kivimaenpaa, M., \& Julkunen-Tiitto, R. (2018). New light for phytochemicals. Trends in Biotechnology, 36(1), 7-10. doi: https://doi.org/10.1016/j.tibtech.2017.08.009 Jaakola, L. \& Hohtola, A. (2010). Effect of latitude on flavonoid biosynthesis in plants. Plant, Cell and Environment, 33(8), 1239-1247.

doi: https://doi.org/10.1111/j.1365-3040.2010.02154.x

Jaakola, L. (2013). New insights into the regulation of anthocyanin biosynthesis in fruits. Trends in Plant Science, 18(9), 477-483. doi: https://doi.org/10.1016/j.tplants.2013.06.003 Jaakola, L., Maatta, K., Pirttila, A. M., Torronen, R., Karenlampi, S., \& Hohtola, A. (2002). Expression of genes involved in anthocyanin biosynthesis in relation to anthocyanin, proanthocyanidin, and flavonol levels during bilberry fruit development. Plant Physiology, 130(2), 729-739. Jaakola, L., Poole, M., Jones, M. O., Kamarainen-Karppinen, T., Koskimaki, J. J., Hohtola, A., Haggman, H., Fraser, P.D., Manning, K., King, G.J., Thomson, H., \& Seymour, G. B. (2010). A SQUAMOSA MADS box gene involved in the regulation of anthocyanin accumulation in bilberry fruits. Plant Physiology, 153(4), 1619-1629. doi:https://doi.org/10.1104/pp.110.158279Jeong, S. T., Goto-Yamamoto, N., Kobayashi, S., \& Esaka, M. (2004). Effects of plant hormones and shading on the accumulation of anthocyanins and the expression of anthocyanin biosynthetic genes in grape berry skins. Plant Science, 167(2), 247-252. doi:https://doi.org/10.1016/j.plantsci.2004.03.021 Jia, H. F., Chai, Y. M., Li, C. L., Lu, D., Luo, J. J., Qin, L., \& Shen, Y. Y. (2011). Abscisic acid plays an important role in the regulation of strawberry fruit ripening. Plant Physiology,157(1), 188-199. doi: https://doi.org/10.1104/pp.111.177311 Kadomura-Ishikawa, Y., Miyawaki, K., Takahashi, A., Masuda, T., \& Noji, S. (2015). Light and abscisic acid independently regulated FaMYB10 inFragaria $x$ ananassa fruit. Planta, 241(4), 953-965. doi: https://doi.org/10.1007/s00425-014-2228-6 Karppinen, K., Lafferty, D., Albert, N., Mikkola, N., McGhie, T., Allan, A., Afzal, B., Haggman, H., Espley, R., Jaakola, L. (2021). MYBA and MYBPA transcription factors co-regulate anthocyanin biosynthesis in blue-coloured berries. submitted to New Phytologist. Karppinen, K., Hirvela, E., Nevala, T., Sipari, N., Suokas, M., \& Jaakola, L. (2013). Changes in the abscisic acid levels and related gene expression during fruit development and ripening in bilberry (Vaccinium myrtillus L.). Phytochemistry, 95, 127-134. doi: https://doi.org/10.1016/j.phytochem.2013.06.023 Karppinen, K., Tegelberg, P., Haggman, H., \& Jaakola, L. (2018). Abscisic acid regulates anthocyanin biosynthesis and gene expression associated with cell wall modification in ripening bilberry (Vaccinium myrtillus L.) fruits. Frontiers in Plant Science, 9, 1-17. doi: https://doi.org/10.3389/fpls.2018.01259 Karppinen, K., Zoratti, L., Nguyenquynh, N., Haggman, H., \& Jaakola, L. (2016a). On the developmental and environmental 
regulation of secondary metabolism in Vaccinium spp. berries. Frontiers in Plant Science, 7, 655. doi: https://doi.org/10.3389/fpls.2016.00655Karppinen, K., Zoratti, L., Sarala, M., Carvalho, E., Hirsimaki, J., Mentula, H., Martens, S., Haggman, H., \& Jaakola, L. (2016b). Carotenoid metabolism during bilberry (Vaccinium myrtillus L.) fruit development under different light conditions is regulated by biosynthesis and degradation. BMC Plant Biology, 16(1), 95. doi: https://doi.org/10.1186/s12870-016-0785-5Kim, S. J., \& Brandizzi, F. (2012). News and views into the SNARE complexity in Arabidopsis. Frontiers in Plant Science, 3, 1-6. doi: https://doi.org/10.3389/fpls.2012.00028 Kokalj, D., Zlatić, E., Cigić, B., Kobav, M. B., \& Vidrih, R. (2019). Postharvest flavonol and anthocyanin accumulation in three apple cultivars in response to bluelight-emitting diode light. Scientia Horticulturae, 257, 108711. Kondo, S., Sugaya, S., Sugawa, S., Ninomiya, M., Kittikorn, M., Okawa, K., Ohara, H., Ueno, K., Todoroki, Y., Mizutani, M., \& Hirai, N. (2012). Dehydration tolerance in apple seedlings is affected by an inhibitor of ABA 8'-hydroxylase CYP707A. Journal of Plant Physiology, 169 (3), 234-241. doi: https://doi.org/10.1016/j.jplph.2011.09.007 Kondo, S., Tomiyama, H., Rodyoung, A., Okawa, K., Ohara, H., Sugaya, S., Terahara, N., \& Hirai, N. (2014). Abscisic acid metabolism and anthocyanin synthesis in grape skin are affected by light emitting diode (LED) irradiation at night. Journal of Plant Physiology,171(10), 823-829. doi: https://doi.org/10.1016/j.jplph.2014.01.001 Kopylova, E., Noé, L., \& Touzet, H. (2012). SortMeRNA: fast and accurate filtering of ribosomal RNAs in metatranscriptomic data. Bioinformatics, 28(24), 3211-3217. doi: https://doi.org/10.1093/bioinformatics/bts611 Koyama, K., Ikeda, H., Poudel, P. R., \& Goto-Yamamoto, N. (2012). Light quality affects flavonoid biosynthesis in young berries of Cabernet Sauvignon grape. Phytochemistry, 78, 54-64. Lau, O. S., \& Deng, X. W. (2012). The photomorphogenic repressors COP1 and DET1: 20 years later. Trends in Plant Science, 17(10), 584-593. doi: https://doi.org/10.1016/j.tplants.2012.05.004Li, G., Zhao, J., Qin, B., Yin, Y., An, W., Mu, Z., \& Cao, Y. (2019). ABA mediates development-dependent anthocyanin biosynthesis and fruit coloration in Lycium plants. BMC Plant Biology,19(1), 1-13. doi:https://doi.org/10.1186/s12870-019-19317Li, Q.-H., \& Yang, H.-Q. (2007). Cryptochrome signaling in plants.Photochemistry and Photobiology, 83(1), 94-101. doi:https://doi.org/10.1562/2006-02-28-ir-826Love, M., Huber, W., \& Anders, S. (2014). Moderated estimation of fold change and dispersion for RNA-seq data with DESeq2. Genome Biology, 15(12). doi:https://doi.org/10.1186/s13059-014-0550-8Lu, X. D., Zhou, C. M., Xu, P. B., Luo, Q., Lian, H. L., \& Yang, H. Q. (2015). Red-light-dependent interaction of phyB with SPA1 promotes COP1SPA1 dissociation and photomorphogenic development in arabidopsis. Molecular Plant, 8(3), 467-478. doi: https://doi.org/10.1016/j.molp.2014.11.025 Ma, Z. H., Li, W. F., Mao, J., Li, W., Zuo, C. W., Zhao, X., Dawuda, M. M., Shi, X. Y., \& Chen, B. H. (2019). Synthesis of light-inducible and light-independent anthocyanins regulated by specific genes in grape "Marselan" ( V. Vinifera L.). PeerJ, 2019(3), 1-24. doi: https://doi.org/10.7717/peerj.6521 Massa, G. D., Kim, H. H., Wheeler, R. M., \& Mitchell, C. A. (2008). Plant productivity in response to LED lighting. HortScience, 43(7), 1951-1956. Miao, L., Zhang, Y., Yang, X., Xiao, J., Zhang, H., Zhang, Z., Wang, Y., \& Jiang, G. (2016). Colored light-quality selective plastic films affect anthocyanin content, enzyme activities, and the expression of flavonoid genes in strawberry (Fragaria $x$ ananassa) fruit.Food Chemistry, 207, 93-100. doi:https://doi.org/10.1016/j.foodchem.2016.02.077Möglich, A., Yang, X., Ayers, R. A., \& Moffat, K. (2010). Structure and function of plant photoreceptors. Annual Review of Plant Biology, 61, 21-47. doi:https://doi.org/10.1146/annurev-arplant-042809-112259Müller, D., Schantz, M., \& Richling, E. (2012). High performance liquid chromatography analysis of anthocyanins in bilberries (Vaccinium myrtillus L.), blueberries (Vaccinium corymbosum L.) and corresponding juices. Journal of Food Science, $77(4)$, C340-C345. Nagaoka, M., Maeda, T., Chatani, M., Handa, K., Yamakawa, T., Kiyohara, S., Negishi-Koga, T., Kato, Y., Takami, M., Niida, S., Lang, S. C., Kruger, M. C., \& Suzuki, K. (2019). A delphinidin-enriched maqui berry extract improves bone metabolism and protects against bone loss in osteopenic mouse models. Antioxidants, 8(9), 386. doi:https://doi.org/10.3390/antiox8090386 Nguyen, N., Suokas, M., Karppinen, K., Vuosku, J., Jaakola, L., \& Häggman, H. (2018). Recognition of candidate transcription factors related to bilberry fruit ripening by de novo transcriptome and qRT-PCR analyses. Scientific Reports, 8(1), 1-12. doi:https://doi.org/10.1038/s41598-018-28158-7Nile, S., \& Park, S. (2014). Edible berries: Bioactive components and their effect on human health. Nutrition, 30(2), 134-144. doi: https://doi.org/10.1016/j.nut.2013.04.007Ouzounis, T., Rosenqvist, E., \& Ottosen, C. O. (2015). Spectral effects of artificial light on plant physiology and secondary metabolism: A review. HortScience, 50(8), 
1128-1135. doi: https://doi.org/10.21273/hortsci.50.8.1128 Park, S.Y., Fung, P., Nishimura, N., Jensen, D. R., Fujii, H., Zhao, Y., ..., \& Cutler, S. R. (2009). Abscisic acid inhibits Type 2C protein phosphatases via the PYR/PYL family of START proteins. Science (New York, N.Y.), 324(5930), 1068-1071. doi:https://doi.org/10.1126/science.1173041Patro, R., Duggal, G., Love, M., Irizarry, R., \& Kingsford, C. (2017). Salmon provides fast and bias-aware quantification of transcript expression. Nature Methods, 14(4), 417-419. doi:https://doi.org/10.1038/nmeth.4197Pečenková, T., Marković, V., Sabol, P., Kulich, I., \& Zárský, V. (2017). Exocyst and autophagy-related membrane trafficking in plants.Journal of Experimental Botany, 69(1), 47-57. doi:https://doi.org/10.1093/jxb/erx363Petrussa, E., Braidot, E., Zancani, M., Peresson, C., Bertolini, A., Patui, S., \& Vianello, A. (2013). Plant flavonoids-biosynthesis, transport and involvement in stress responses. International Journal of Molecular Sciences, 14 (7), 14950-14973. doi: https://doi.org/10.3390/ijms140714950 Plunkett, B.J., Espley, R.V., Dare, A.P., Warren, B.A.W., Grierson, E.R.P., Cordiner, S., Turner, J.L., Allan, A.C., Albert, N.W., Davies, K.M., Schwinn, K.E. (2018). MYBA from blueberry (Vacciniumsection Cyanococcus) is a subgroup 6 type R2R3MYB transcription factor that activates anthocyanin production. Frontiers in Plant Science 9: 1300. Primetta, A. K., Karppinen, K., Riihinen, K. R., \& Jaakola, L. (2015). Metabolic and molecular analyses of white mutant Vaccinium berries show down-regulation of MYBPA1-type R2R3 MYB regulatory factor. Planta, 242(3), 631-643. doi:https://doi.org/10.1007/s00425-015-2363-8Ravaglia, D., Espley, R.V., Henry-Kirk R.A., Andreotti, C., Ziosi, V., Hellens, R.P., Costa, G., \& Allan, A.C. (2013). Transcriptional regulation of flavonoid biosynthesis in nectarine (Prunus persica) by a set of R2R3 MYB transcription factors. BMC Plant Biology 13, 68. Rodyoung, A., Masuda, Y., Tomiyama, H., Saito, T., Okawa, K., Ohara, H., \& Kondo, S. (2016). Effects of light emitting diode irradiation at night on abscisic acid metabolism and anthocyanin synthesis in grapes in different growing seasons. Plant Growth Regulation, 79(1), 39-46. doi: https://doi.org/10.1007/s10725-0150107-1Simão, F. A., Waterhouse, R. M., Ioannidis, P., Kriventseva, E. V., \& Zdobnov, E. M. (2015). BUSCO: assessing genome assembly and annotation completeness with single-copy orthologs. Bioinformatics (Oxford, England), 31 (19), 3210-3212. doi: https://doi.org/10.1093/bioinformatics/btv351 Takos, A.M., Jaffé, F.W., Jacob, S.R., Bogs, J., Robinson, S.P., \& Walker, A.R. (2006). Light-induced expression of a MYB gene regulates anthocyanin biosynthesis in red apples. Plant Physiology 142, 1216-1232. Tao, R., Bai, S., Ni, J., Yang, Q., Zhao, Y., \& Teng, Y. (2018). The blue light signal transduction pathway is involved in anthocyanin accumulation in 'Red Zaosu' pear. Planta, 248(1), 37-48. Thornthwaite, J. T., Thibado, S. P., \& Thornthwaite, K. A. (2020). Bilberry anthocyanins as agents to address oxidative stress.Pathology. INC, 179-187. doi: https://doi.org/10.1016/b978-0-12-815972-9.00017-2 Tohge T, de Souza LP, Fernie AR. (2017). Current understanding of the pathways of flavonoid biosynthesis in model and crop plants. Journal of Experimental Botany 68: 4013-4028. Walker, A. R., Lee, E., Bogs, J., McDavid, D. A. J., Thomas, M. R., \& Robinson, S. P. (2007). White grapes arose through the mutation of two similar and adjacent regulatory genes. Plant Journal,49(5), 772-785. doi: https://doi.org/10.1111/j.1365-313X.2006.02997.x Wang, J. W., Czech, B., \& Weigel, D. (2009). miR156-Regulated SPL transcription factors define an endogenous flowering pathway inArabidopsis thaliana. Cell, 138(4), 738-749. doi:https://doi.org/10.1016/j.cell.2009.06.014Wheeler, S., Loveys, B., Ford, C., \& Davies, C. (2009). The relationship between the expression of abscisic acid biosynthesis genes, accumulation of abscisic acid and the promotion of Vitis viniferaL. berry ripening by abscisic acid. Australian Journal of Grape and Wine Research, 15(3), 195-204. doi: https://doi.org/10.1111/j.17550238.2008.00045.x Wu, C., Deng, C., Hilario, E., Albert, N., Lafferty, D., Grierson, E., ..., Chagne, D. (2021). A chromosome-scale assembly of the bilberry genome identifies a complex locus controlling berry anthocyanin composition". submitted to Molecular Ecology Resources. Wu, J., Mao, X., Cai, T., Luo, J., \& Wei, L. (2006). KOBAS server: a web-based platform for automated annotation and pathway identification. Nucleic Acids Research, 34(Web Server), W720-W724. doi: https://doi.org/10.1093/nar/gkl167Wu, M., Si, M., Li, X., Song, L., Liu, J., Zhai, R., Cong, L., Yue, R., Yang, C., Ma, F., Xu, L., \& Wang, Z. (2019). PbCOP1.1 contributes to the negative regulation of anthocyanin biosynthesis in pear.Plants, 8(2), 1-12. doi: https://doi.org/10.3390/plants8020039 Wu, X., Gong, Q., Ni, X., Zhou, Y., \& Gao, Z. (2017). UFGT: The key enzyme associated with the petals variegation in japanese apricot.Frontiers in Plant Science, 8, 108.doi: https://doi.org/10.3389/fpls.2017.00108 Xu, W., Dubos, C., \& Lepiniec, L. (2015). Transcriptional control of flavonoid biosynthesis by MYB-bHLH-WDR complexes. Trends in Plant Sci- 
ence, 20(3), 176-185. Yadav, V., Mallappa, C., Gangappa, S. N., Bhatia, S., \& Chattopadhyay, S. (2005). A basic helix-loop-helix transcription factor in Arabidopsis, MYC2, acts as a repressor of blue light-mediated photomorphogenic growth. The Plant Cell, 17(7), 1953-1966. doi: https://doi.org/10.1105/tpc.105.032060 Zhang, Y., Jiang, L., Li, Y., Chen, Q., Ye, Y., Zhang, Y., Luo, Y., Sun, B., Wang, X., \& Tang, H. (2018). Effect of red and blue light on anthocyanin accumulation and differential gene expression in strawberry (Fragaria $x$ ananassa). Molecules, 23(4), 1-17. doi: https://doi.org/10.3390/molecules23040820 Zhang, J., Zhang, Y., Song, S., Su, W., Hao, Y., \& Liu, H. (2020). Supplementary red light results in the earlier ripening of tomato fruit depending on ethylene production. Environmental and Experimental Botany, 175, 104044. doi:https://doi.org/10.1016/j.envexpbot.2020.104044Zhao, J. (2015). Flavonoid transport mechanisms: How to go, and with whom. Trends in Plant Science, 20(9), 576-585. doi:https://doi.org/10.1016/j.tplants.2015.06.007Zoratti, L., Karppinen, K., Luengo Escobar, A., Haggman, H., \& Jaakola, L. (2014a). Light-controlled flavonoid biosynthesis in fruits. Frontiers in Plant Science, 5, 534. doi: https://doi.org/10.3389/fpls.2014.00534 Zoratti, L., Sarala, M., Carvalho, E., Karppinen, K., Martens, S., Giongo, L., Haggman, H., and Jaakola, L. (2014b) Monochromatic light increases anthocyanin content during fruit development in bilberry. BMC Plant Biol. 14:377. doi: https://doi.org/ 10.1186/s12870-0140377-1Zoratti, L., Klemettila, H., \& Jaakola, L. (2016). Bilberry (Vaccinium myrtillus L.) Ecotypes. Nutritional Composition of Fruit Cultivars, 83-99. doi: https://doi.org/10.1016/b978-0-12-408117-8.0000 Zorenc Z., Veberic, R., Slatnar, A., Koron, D., Miosic, S., Chen, M.H., Haselmair-Gosch, C., Halbwirth, H., MikulicPetkovsek, M. (2017). A wild 'albino' bilberry (Vaccinium myrtillus L.) from Slovenia shows three bottlenecks in the anthocyanin pathway and significant differences in the expression of several regulatory genes compared to the common blue berry type. PLoS One 12: e0190246.

Tables

Table 1 . Statistics of combined Trinity transcriptome assembly after concatenating de novo and genomeguided assemblies.

\begin{tabular}{lll}
\hline Assembly & Transcripts & Unigenes \\
\hline Total counts & 671,952 & 472,876 \\
Contig N50 (bp) & 1567 & 1122 \\
Contig N30 (bp) & 2431 & 2035 \\
Average contig length (bp) & 910.78 & 720.23 \\
Median contig length (bp) & 508 & 408 \\
Total assembled bases & $611,998,789$ & $340,578,754$ \\
Average read length 150 bp & & \\
Percent GC 41.71\% & & \\
\hline
\end{tabular}

Figure legends

Figure 1. Spectral light treatments of bilberry plants.

(a ) Supplemental blue $(460 \mathrm{~nm})$ and red $(660 \mathrm{~nm})$ light treatments provided by Heliospectra LED lamps. (b) Developmental stages of bilberry: S1, flower; S2, small unripe green fruit; S3, large unripe green fruit; S4, ripening purple fruit; and S5, fully ripe blue fruit. (c) Light spectra and relative light intensities in the light treatments expressed in arbitrary units (AU).

\section{Figure 2. Functional annotation of bilberry transcriptome}

(a ) Summary of functional annotation from Trinotate pipeline. x-axis denotes the number of hits from the unigenes and y-axis denotes the different databases utilized for search. (b ) Co-expression of genes among red, blue, and control light treatments represented as venn-diagram.

Figure 3. Differential gene expression analysis from the light treated bilberry samples 
(a ) Clustered hierarchical heat map of normalized differential gene expression among the samples $(\mathrm{H}-$ cluster). The scale bars from -2 to 2 represent the $\log 2$ values of fragments per kilobase of transcript per million mapped reads (FPKM). (b ) volcano plot of red vs control comparison. (c) Volcano plot of blue vs control comparison. Both the contrasts were FDR corrected / p-value adjusted to [?] 0.05 for obtaining DEGs and plotted with the $\log 2$ fold changes against the adjusted - $\log _{10} \mathrm{p}$-values obtained.

\section{Figure 4. Enrichment analysis of Gene Ontology (GO) terms and KEGG pathways}

(a ) The number of significantly enriched GO terms obtained against the top 500 DEG sequences. The treatment contrasts are represented with similar colors. The GO terms are categorized into Biological process (BP), Molecular function (MF) and Cellular process (CC). (b ) Number of enriched GO terms obtained from direct count of sequences in Biological process (BP) category. (c )The KEGG metabolic pathways significantly enriched in red vs control and (d ) blue vs control. corresponding circle sizes represent the counts of sequences with p-value $<0.01$.

Figure 5. DEGs from light signaling and circadian rhythm. Schematic representation of red and blue light signal perception by higher plants which are likely to be involved in light regulated anthocyanin biosynthesis. The associated DEGs from red and blue light vs control contrasts were represented in two color code boxes based on $\log 2$ fold changes. Red light treatment is shown left and blue light treatment on the right side of the box.Abbreviations and gene definitions: PHYA/B, Phytochrome; CRY1/2, Cryptochrome; COP1, constitutive photomorphogenic; Elf3, early flowering 3; FT, flowering locus T; PRR5, pseudo response regulator 5; CK2 $\alpha$, casein kinase II subunit alpha; Cab, chlorophyll a/b binding protein; Exp, expansin; HY5, elongated hypocotyl; FKF1- flavin-binding kelch domain F box protein; SPA, suppressor of PhyA; HY5, elongated hypocotyl 5; CDF1, cycling DOF factor 1; Co, Constans.

Figure 6. DEGs from anthocyanin, carotenoid and ABA biosynthesis. Schematic representation of bilberry anthocyanin biosynthetic pathway branching from phenylpropanoid biosynthesis (top) and representation of carotenoid biosynthetic pathway leading towards abscisic acid (ABA) biosynthesis and catabolism. DEGs from flavonoid, carotenoid-ABA pathway genes and selected TFs visualized as heatmap based on $\log 2$ fold changes obtained from light treatments against the control samples. Enzyme abbreviations: PAL, phenylalanine ammonia-lyase; $\mathrm{C} 4 \mathrm{H}$, cinnamate 4-hydroxylase; 4CL, 4-coumarate:CoA ligase; CCoAOMT, caffeoyl-CoA O-methyltransferase; Prx,cPrx- peroxidases, cationic peroxidases; CHS, chalcone synthase; CHI, chalcone isomerase; F3H, flavanone 3-hydroxylase; F3'H, flavonoid 3' hydroxylase; F3'5'H, flavonoid 3'5' hydroxylase; DFR, dihydroflavonol 4-reductase; ANS, anthocyanidin synthase; UFGT, UDPglucose flavonoid 3- $O$-glucosyltransferase; PSY, phytoene synthase; ZDS, zeta-carotene desaturase; $\mathrm{BCH}$, beta-carotene hydroxylase; CYP 450-BCH, carotenoid $\beta$-ring hydroxylase of cytochrome P450 family; LUT1, lutein deficient 1; NCED, 9-cis -epoxycarotenoid dioxygenase; ABA 8' hyd, Abscisic acid 8' hydroxylase, PYR/PYL, Pyrabactin-resistance like.

Figure 7. DEGs from SNARE mediated vesicular trafficking.Schematic representation of vesicular transport of anthocyanins mediated by SNARE proteins interaction through transmembrane - Endoplasmic reticulum (ER) - golgi network. The associated transporter genes from red and blue light vs control contrasts DEGs were represented in color code boxes based on log2 fold changes. Red light treatment is shown left and blue light treatment on the right side of the box.Abbreviations and gene definitions: Stx, syntaxin-like; Bos1, Gos, Qb type golgi SNAP receptor complex; Ykt6, Sec22, vesicle transporter/VAMP like protein; SNAP, soluble NSF attachment protein; SNARE, 'SNAP REceptor'.

\section{Figure 8. Quantitative determination of anthocyanin content}

(a) Anthocyanin content from light treated (6-day) bilberry samples determined through LC-MS. The relative content expressed in arbitrary units (AU) and was calculated by normalizing the analyte peaks area with the dry weight of the samples $\left(\mathrm{AU} \mathrm{mg}^{-1} \mathrm{DW}\right)$. Del-Delphinidins, Cyn- Cyanidins, Pet-Petunidins, MvMalvidins; gal-galactoside, glu-glucoside, ara-arabinoside. (b) Total anthocyanins expressed in $\mathrm{mg} \mathrm{g}^{-1} \mathrm{FW}$ of cyanidin 3-glucoside equivalents after 12 days of spectral light treatment. Different letters denote significant differences among groups analyzed by one-way ANOVA followed by Turkey's post hoc test (p-value $<0,05)$. 
Figure 9. Metabolite-gene expression correlation analysis associated with anthocyanin, carotenoid and ABA metabolism

Pearson's coefficient-based correlation matrices from selected gene expression levels with two major identified delphinidin glycosides for (a ) control vs red (b ) control vs blue contrasts. The size and color of dots represents the strength of correlation from positive (blue) to negative (red) correlations arranged in an upper triangular matrix.

(a)

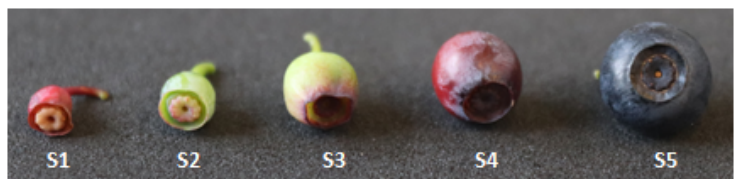

(b)

(c)
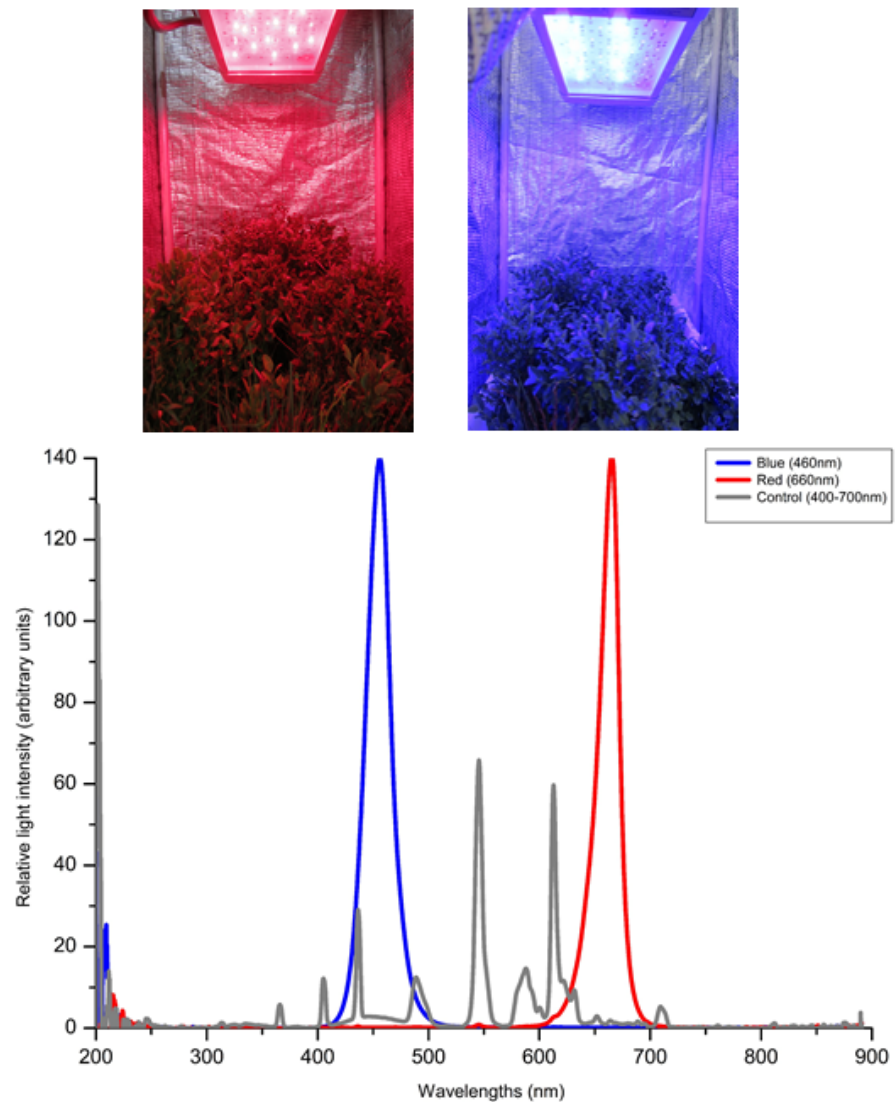

Figure 1. Spectral light treatments of bilberry plants.

(a ) Supplemental blue $(460 \mathrm{~nm})$ and red $(660 \mathrm{~nm})$ light treatments provided by Heliospectra LED lamps. (b) Developmental stages of bilberry: S1, flower; S2, small unripe green fruit; S3, large unripe green fruit; S4, ripening purple fruit; and S5, fully ripe blue fruit. (c ) Light spectra and relative light intensities in the light treatments expressed in arbitrary units (AU). 
(a)

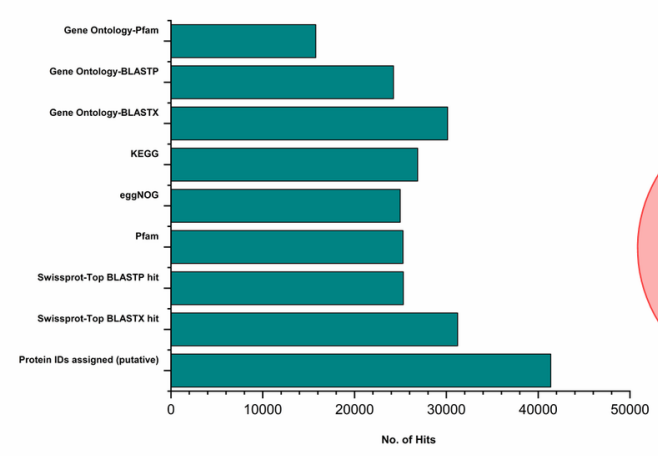

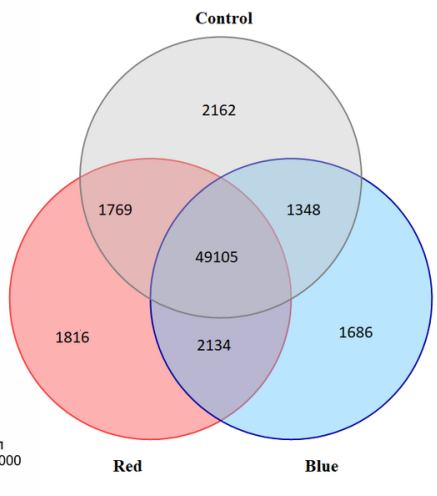

Figure 2. Functional annotation of bilberry transcriptome

(a ) Summary of functional annotation from Trinotate pipeline. x-axis denotes the number of hits from the unigenes and y-axis denotes the different databases utilized for search. (b ) Co-expression of genes among red, blue, and control light treatments represented as venn-diagram.
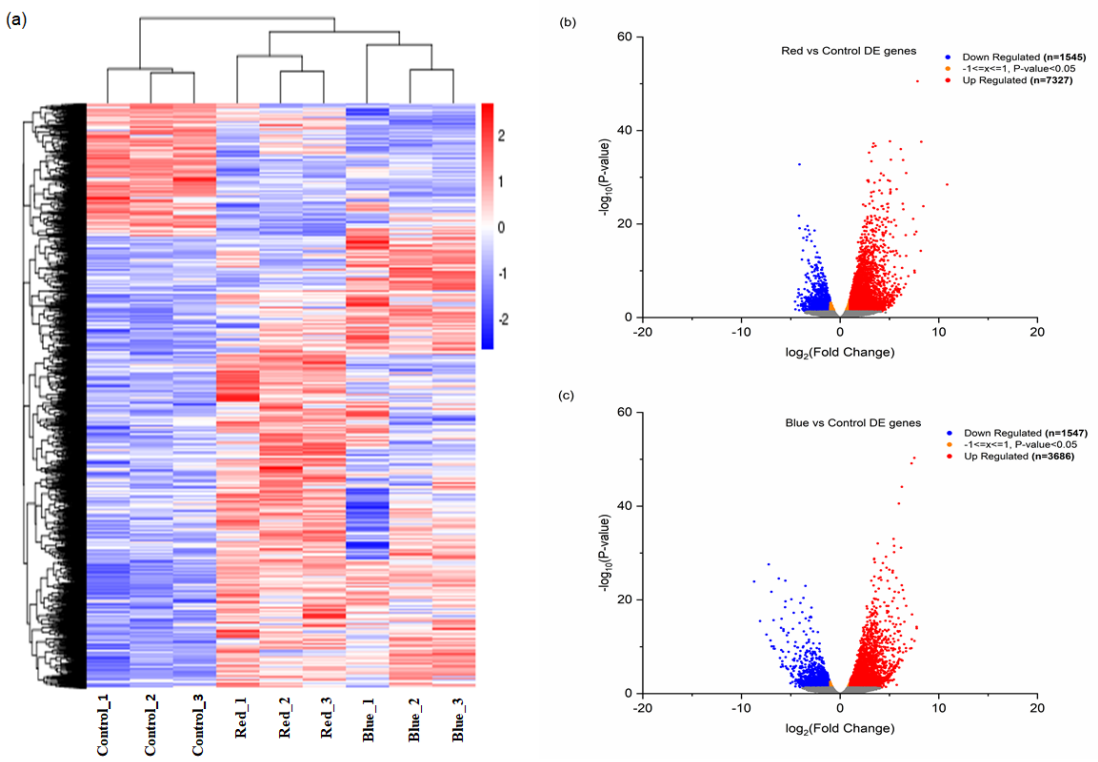

\section{Figure 3. Differential gene expression analysis from the light treated bilberry samples}

(a ) Clustered hierarchical heat map of normalized differential gene expression among the samples $(\mathrm{H}-$ cluster). The scale bars from -2 to 2 represent the $\log 2$ values of fragments per kilobase of transcript per million mapped reads (FPKM). (b ) volcano plot of red vs control comparison. (c ) Volcano plot of blue vs control comparison. Both the contrasts were FDR corrected / p-value adjusted to [?] 0.05 for obtaining DEGs and plotted with the $\log 2$ fold changes against the adjusted - $\log _{10}$ p-values obtained. 

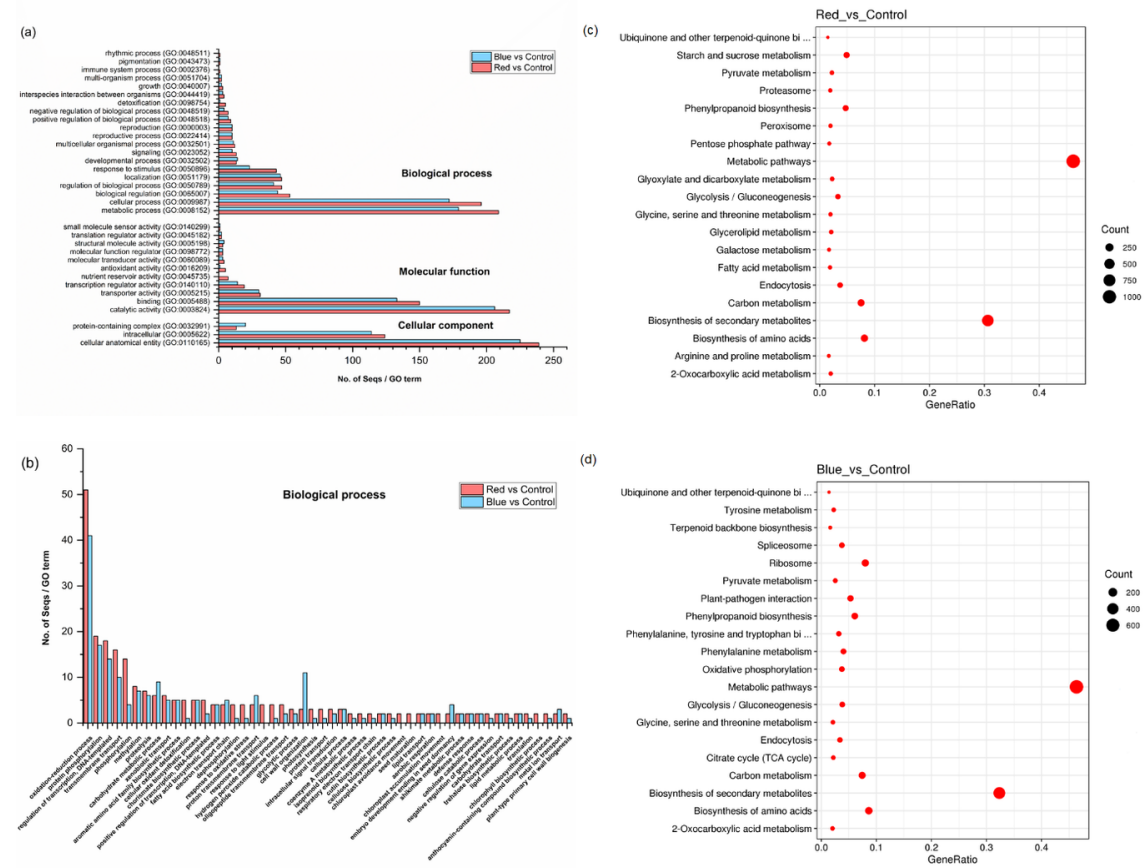

\section{Figure 4. Enrichment analysis of Gene Ontology (GO) terms and KEGG pathways}

(a ) The number of significantly enriched GO terms obtained against the top 500 DEG sequences. The treatment contrasts are represented with similar colors. The GO terms are categorized into Biological process (BP), Molecular function (MF) and Cellular process (CC). (b ) Number of enriched GO terms obtained from direct count of sequences in Biological process (BP) category. (c )The KEGG metabolic pathways significantly enriched in red vs control and (d ) blue vs control. corresponding circle sizes represent the counts of sequences with p-value $<0.01$. 


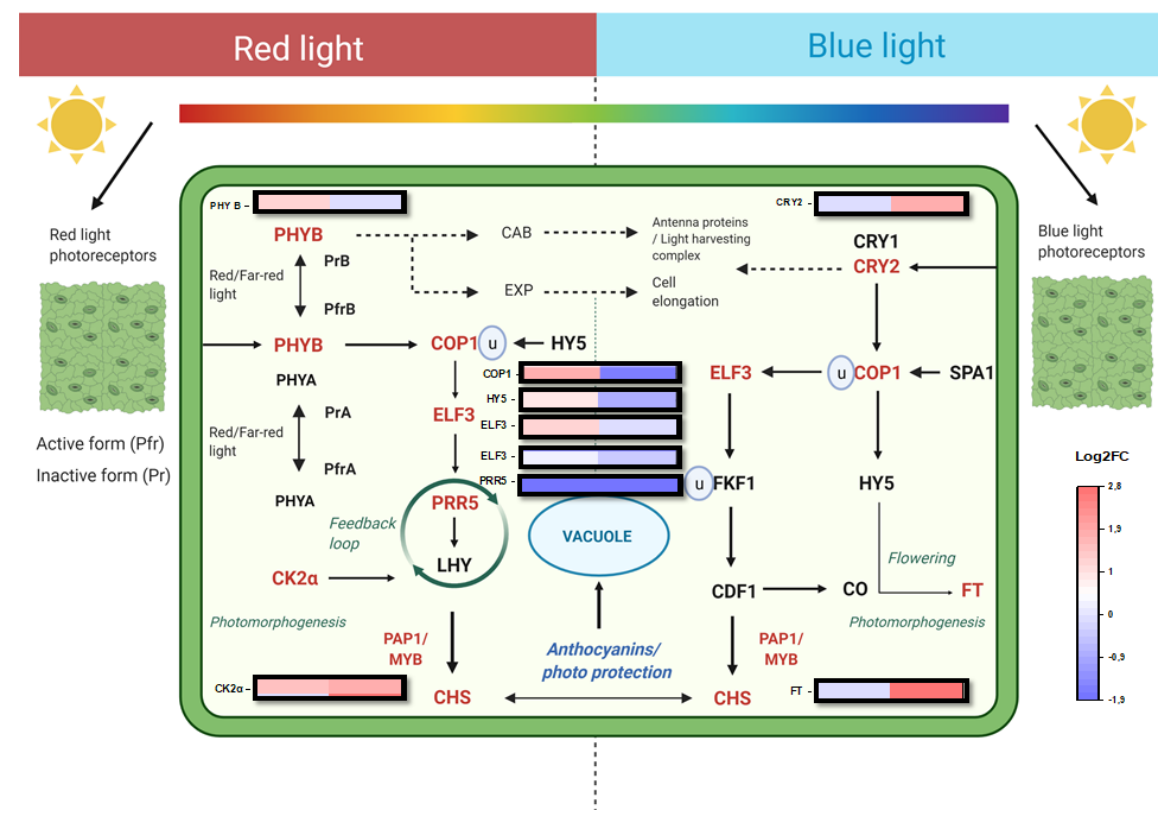

Figure 5. DEGs from light signaling and circadian rhythm. Schematic representation of red and blue light signal perception by higher plants which are likely to be involved in light regulated anthocyanin biosynthesis. The associated DEGs from red and blue light vs control contrasts were represented in two color code boxes based on $\log 2$ fold changes. Red light treatment is shown left and blue light treatment on the right side of the box.Abbreviations and gene definitions: PHYA/B, Phytochrome; CRY1/2, Cryptochrome; COP1, constitutive photomorphogenic; Elf3, early flowering 3; FT, flowering locus T; PRR5, pseudo response regulator 5; CK2 $\alpha$, casein kinase II subunit alpha; Cab, chlorophyll a/b binding protein; Exp, expansin; HY5, elongated hypocotyl; FKF1- flavin-binding kelch domain F box protein; SPA, suppressor of PhyA; HY5, elongated hypocotyl 5; CDF1, cycling DOF factor 1; Co, Constans.
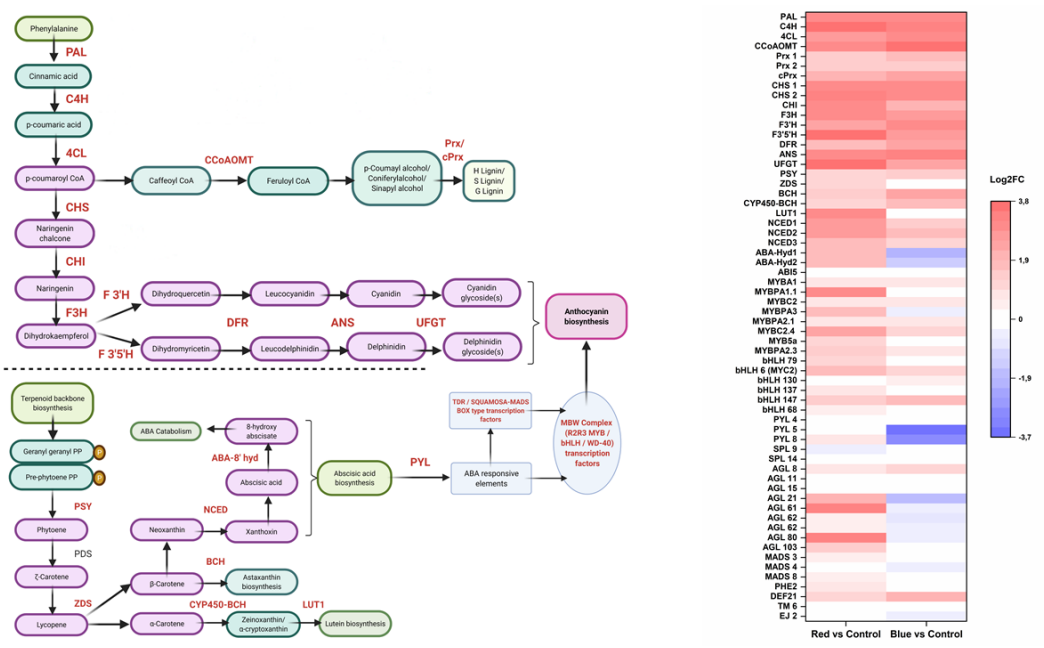

Figure 6. DEGs from anthocyanin, carotenoid and ABA biosynthesis. Schematic representation of bilberry anthocyanin biosynthetic pathway branching from phenylpropanoid biosynthesis (top) and representation of carotenoid biosynthetic pathway leading towards abscisic acid (ABA) biosynthesis and catabolism. DEGs from flavonoid, carotenoid-ABA pathway genes and selected TFs visualized as heatmap 
based on $\log 2$ fold changes obtained from light treatments against the control samples. Enzyme abbreviations: PAL, phenylalanine ammonia-lyase; $\mathrm{C} 4 \mathrm{H}$, cinnamate 4-hydroxylase; 4CL, 4-coumarate:CoA ligase; CCoAOMT, caffeoyl-CoA O-methyltransferase; Prx,cPrx- peroxidases, cationic peroxidases; CHS, chalcone synthase; CHI, chalcone isomerase; F3H, flavanone 3-hydroxylase; F3'H, flavonoid 3' hydroxylase; F3'5'H, flavonoid 3'5' hydroxylase; DFR, dihydroflavonol 4-reductase; ANS, anthocyanidin synthase; UFGT, UDPglucose flavonoid 3- $O$-glucosyltransferase; PSY, phytoene synthase; ZDS, zeta-carotene desaturase; $\mathrm{BCH}$, beta-carotene hydroxylase; CYP 450-BCH, carotenoid $\beta$-ring hydroxylase of cytochrome P450 family; LUT1, lutein deficient 1; NCED, 9-cis -epoxycarotenoid dioxygenase; ABA 8' hyd, Abscisic acid 8' hydroxylase, PYR/PYL, Pyrabactin-resistance like.

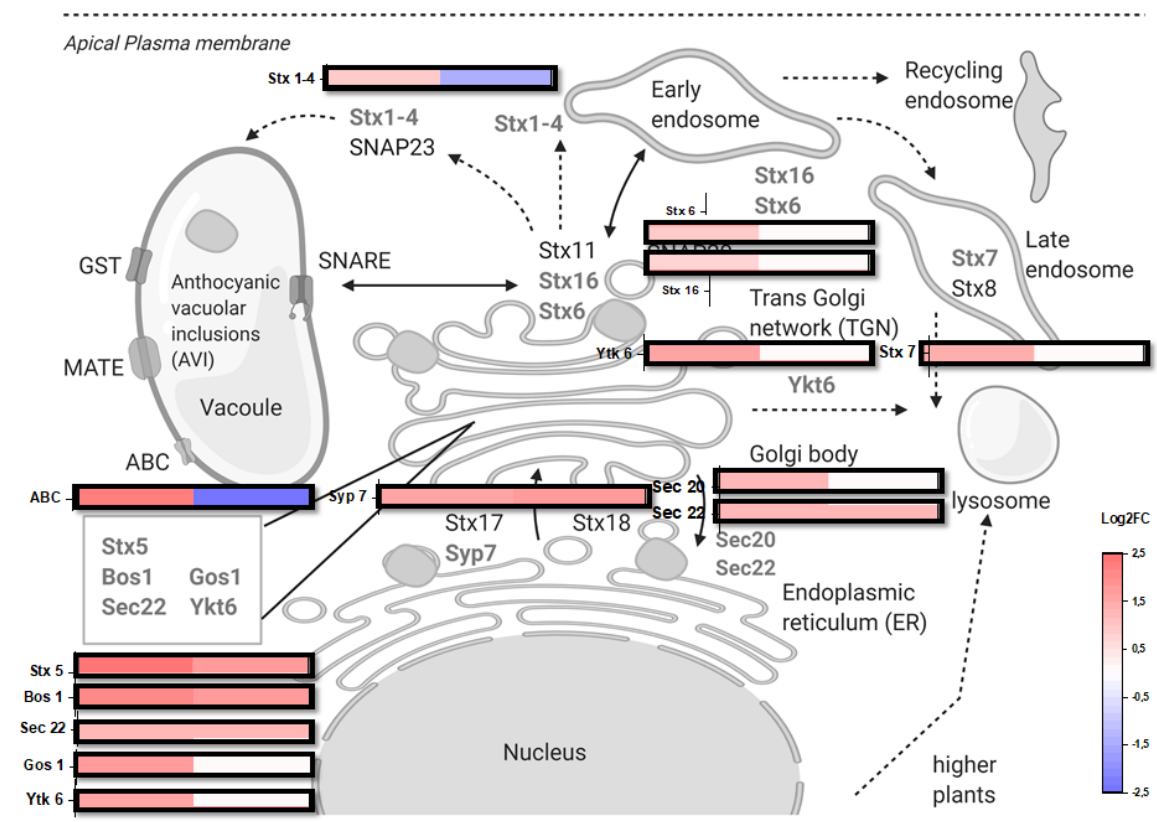

Figure 7. DEGs from SNARE mediated vesicular trafficking.Schematic representation of vesicular transport of anthocyanins mediated by SNARE proteins interaction through transmembrane - Endoplasmic reticulum (ER) - golgi network. The associated transporter genes from red and blue light vs control contrasts DEGs were represented in color code boxes based on $\log 2$ fold changes. Red light treatment is shown left and blue light treatment on the right side of the box.Abbreviations and gene definitions: Stx, syntaxin-like; Bos1, Gos, Qb type golgi SNAP receptor complex; Ykt6, Sec22, vesicle transporter/VAMP like protein; SNAP, soluble NSF attachment protein; SNARE, 'SNAP REceptor'. 

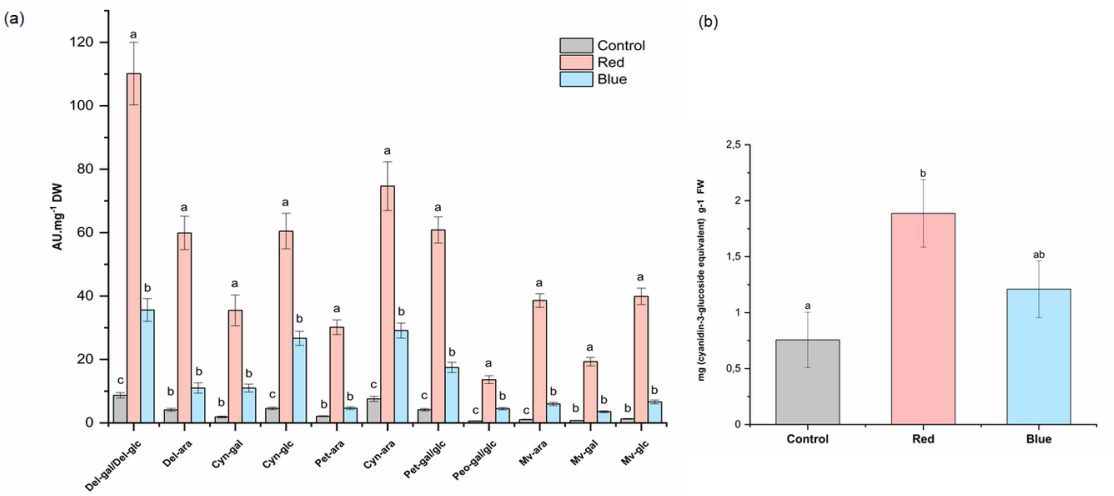

Figure 8. Quantitative determination of anthocyanin content

(a) Anthocyanin content from light treated (6-day) bilberry samples determined through LC-MS. The relative content expressed in arbitrary units (AU) and was calculated by normalizing the analyte peaks area with the dry weight of the samples $\left(\mathrm{AU} \mathrm{mg}^{-1} \mathrm{DW}\right)$. Del-Delphinidins, Cyn- Cyanidins, Pet-Petunidins, MvMalvidins; gal-galactoside, glu-glucoside, ara-arabinoside. (b) Total anthocyanins expressed in $\mathrm{mg} \mathrm{g}^{-1} \mathrm{FW}$ of cyanidin 3-glucoside equivalents after 12 days of spectral light treatment. Different letters denote significant differences among groups analyzed by one-way ANOVA followed by Turkey's post hoc test ( $\mathrm{p}$-value $<0,05)$.
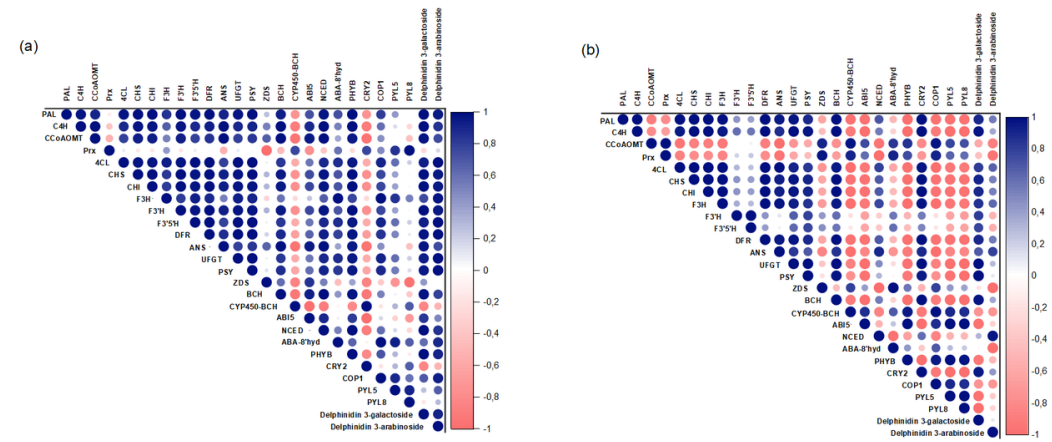

Figure 9. Metabolite-gene expression correlation analysis associated with anthocyanin, carotenoid and ABA metabolism

Pearson's coefficient-based correlation matrices from selected gene expression levels with two major identified delphinidin glycosides for (a ) control vs red (b ) control vs blue contrasts. The size and color of dots represents the strength of correlation from positive (blue) to negative (red) correlations arranged in an upper triangular matrix. 